\title{
Weisungen bei bedingtem Strafvollzug und deren Verhältnis zu Massnahmen
}

\section{Luca Ranzoni *}

Strafrechtliche Weisungen im Zusammenhang mit bedingten Strafen bieten selten Anlass zur Diskussion. Sie sind seit jeher präsent, unbemerkt, fast vergessen. Dass das Weisungsinstitut von einem theoretischen Standpunkt nicht hinreichend durchdacht ist, zeigt sich an verschiedenen Stellen, nicht zuletzt in der oft unklaren Abgrenzung zu strafrechtlichen Massnahmen. Dieser Artikel soll einen Beitrag zur Klärung der Verhältnisse leisten.

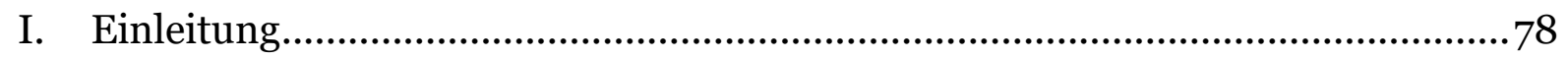

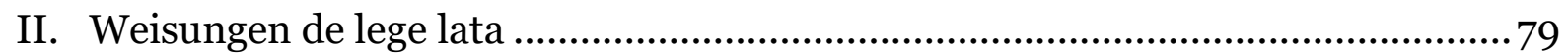

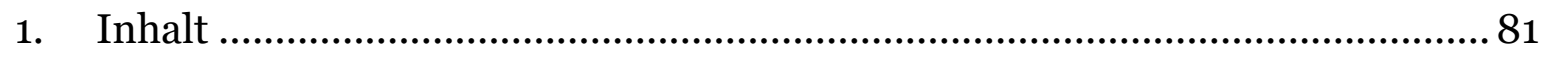

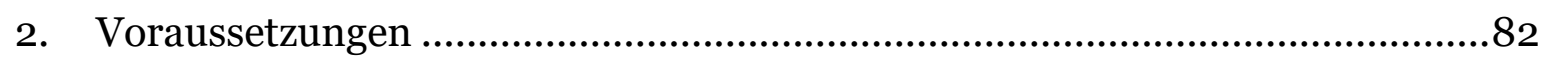

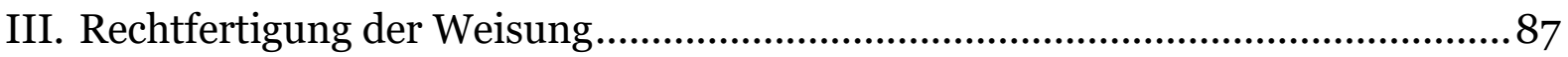

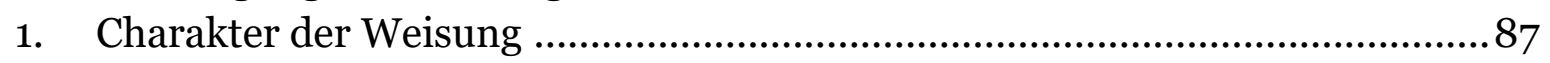

2. Charakter der bedingten Strafe ....................................................... 88

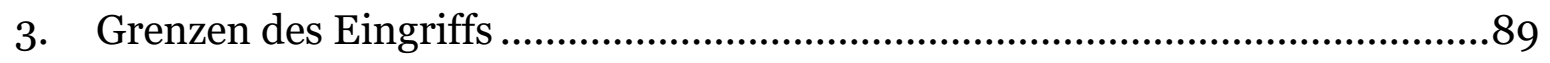

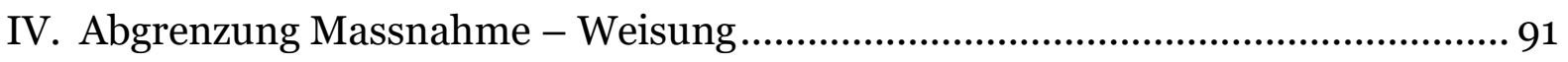

1. Ambulante Massnahme vs. Weisung «Betreuung» ..................................... 91

2. Tätigkeitsverbot vs. Weisung «Berufsausübung» .......................................97

3. Kontakt- und Rayonverbot vs. Weisung «Aufenthalt» .............................. 101

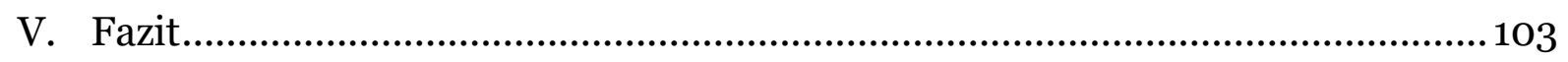

Zitiervorschlag: Luca Ranzoni, Weisungen bei bedingtem Strafvollzug und deren Verhältnis zu Massnahmen, in: sui-generis 2018, S. 77

URL: $\quad$ sui-generis.ch/60

DOI: $\quad$ https://doi.org/10.21257/sg.6o

* Luca Ranzoni, cand. MLaw, ist Assistent am Lehrstuhl von Prof. Dr. iur. Marc Thommen an der Universität Zürich und befindet sich aktuell im LL.M.-Studium in Maastricht (NL). Der vorliegende Aufsatz entspricht einer gekürzten und überarbeiteten Version der mit dem Semesterpreis der Universität Zürich ausgezeichneten Masterarbeit des Autors. Er dankt Prof. Dr. iur. Gunhild Godenzi, MLaw Peter Frick und MLaw Moritz Oehen für ihre Unterstützung. 


\section{Einleitung}

1 «Die Weisungen, welche das Gericht oder die Strafvollzugsbehörde dem Verurteilten für die Probezeit erteilen kann, betreffen insbesondere die Berufsausübung, den Aufenthalt, das Führen eines Motorfahrzeuges, den Schadenersatz sowie die ärztliche und psychologische Betreuung.»1

2 Diese Weisungsbestimmung hat über sämtliche Revisionen des Strafgesetzbuches hinweg nur marginale Änderungen erfahren. Parallel ist der Sanktionskatalog jedoch stark erweitert und umgestaltet worden, ohne dass die Weisungskompetenz eingeschränkt oder ihr Anwendungsbereich präzisiert wurde.

Un nur ein paar Beispiele zu nennen: Die Möglichkeit der ambulant vollzogenen therapeutischen Massnahme wurde erst mit der Revision des Strafgesetzbuches $1971^{2}$ eingeführt.3 Der Bundesrat hatte sich damals dahingehend geäussert, dass es für den bedingten Strafvollzug keiner spezifischen Regelung der ambulanten Massnahme bedürfe, da die Behandlung dort mittels Weisung möglich sei.4 Begründet wurde diese Feststellung nicht.
4 Im Zuge der Revision des Allgemeinen Teils des Strafgesetzbuches von 20025 hat die vorberatende Expertenkommission $^{6}$ dann den bis heute letzten Versuch einer Klärung unternommen. In den Protokollen findet sich eine Fülle von Ideen, die von der Abschaffung der Weisungskompetenz ${ }^{7}$ bis zur Streichung der Widerrufsmöglichkeit und Ersetzung durch einen Verweis auf Art. 292 StGB reichen. ${ }^{8}$ In die schlussendlich in Rechtskraft erwachsene Norm (Art. 94 StGB) wurde davon jedoch kaum etwas übernommen, sodass die Bestimmung weitgehend der ursprünglichen Lösung entspricht. 9

5 Referendumsvorlage vom 13. Dezember $2002 \mathrm{zu}$ den Änderungen des Schweizerischen Strafgesetzbuches (BBl 2002 8240), (fortan: StGB/ 2002).

6 Protokolle der Sitzungen der Subkommission «Sanktionen» der Expertenkommission für die Revision des Allgemeinen Teils, Bern 1995 (fortan: Protokolle, Expertenkommission). Bedingung des Bundesamts für Justiz für die Einsichtnahme in die nicht-öffentlichen Protokolle ist der Verzicht auf wörtliches Zitieren und Nennung der Votanten. Die Lektüre ist sehr zu empfehlen.

7 So Protokolle, Expertenkommission (Fn. 6), 442, 616 sowie 1069.

8 Vorentwurf der Expertenkommission zum Allgemeinen Teil und zum Dritten Buch des Strafgesetzbuches und zu einem Bundesgesetz über die Jugendstrafrechtspflege, Bern 1993 (fortan: VEStGB/1993), Art. 37 Abs. 2; vgl. Bericht zur Revision des Allgemeinen Teils und des Dritten Buches des Strafgesetzbuches und zu einem Bundesgesetz über die Jugendstrafrechtpflege, Erstellt auf der Grundlage der Schlussberichte der Expertenkommision, Bern 1993 (fortan: Bericht VE-StGB/1993), 53 f.; vgl. auch Protokolle, Expertenkommission (Fn. 6), $473 \mathrm{ff}$.

9 Die vom Bundesrat gewählte Formulierung deckt sich auch mehrheitlich bereits mit dem Vorschlag in Hans Schultz, Vorentwurf zur Revision des Allgemeinen Teil und des Dritten Buches «Einführung und Anwendung des Gesetzes» des Schweizerischen Strafgesetzbuches, Bern 1987 (fortan: VE-Schultz), Art. 57 Ziff. 2; vgl. auch AB 1999 S 1134; AB 2001 N 589; Botschaft vom 21. September 1998 zur Änderung des Schweizerischen Strafgesetzbuches (Allgemeine Bestimmungen, Einführung und Anwendung des Gesetzes) und des Militärstrafgesetzes sowie zu einem 

(teil-)bedingte und die unbedingte Geldstrafe eingeführt. ${ }^{10}$ Für die bedingte Geld- und Freiheitsstrafe wurde eine gemeinsame Bestimmung geschaffen (Art. 44 Abs. 2 StGB), welche dem Gericht die Erteilung von Weisungen nach Art. 94 StGB ermöglicht. Der Weisungsmöglichkeit bei einer Probezeit war man sich dabei wohl bewusst, die Möglichkeit der Kombination mit Geldstrafen wurde aber - soweit ersichtlich - zu keinem Zeitpunkt diskutiert.11

6 Somit besteht heute eine Weisungsbestimmung, deren Anwendungsbereich unklar ist. Abgrenzungsschwierigkeiten zwischen Massnahmen und Weisungen stellen sich dabei primär dem Gericht, da sich dieses im Einzelfall zwischen Massnahme und Weisung «entscheiden» muss. Die Betrachtung beschränkt sich deshalb auf gerichtliche Weisungen bei bedingtem Strafvollzug.

\section{Weisungen de lege lata}

7 Weisungen können dem Verurteilten für die Probezeit erteilt werden (Art. 94 StGB). Wird der Vollzug einer Strafe aufgeschoben, muss das Gericht eine Probezeit von zwei bis fünf Jahren festsetzen (Art. 44 Abs. 1 StGB). Zusätzlich kann das Gericht Weisungen erteilen (Art. 44 Abs. 2 StGB). ${ }^{12}$

Bundesgesetz über das Jugendstrafrecht (BBl 1999 II 1979).

10 Art. 34 und $42 \mathrm{StGB} / 2002$ (Fn. 5).

11 Vgl. Botschaft, BBl 1999 II 1979 (Fn. 9), 2042, 2045 f., 2054 zum Institut des «Aussetzen[s] der Strafe», Art. 42 dort; dieses wurde aber im Parlament verworfen, siehe AB $1999 \mathrm{~S} 1116 \mathrm{f}$. Nicht angesprochen wurden die Weisungen im $\mathrm{Zu}-$ sammenhang mit Geldstrafen, vgl. AB 1999 S 1118 ff., 1134 sowie AB 2001 N 556.

12 Roland M. Schneider/Roy Garré, Art. 44, in: Marcel A. Niggli/Hans Wiprächtiger (Hrsg.), Bas-
8 Form: Weisungen sind im Urteil 13 festzuhalten und zu begründen (Art. 95 Abs. 2 StGB). Der Weisungsinhalt muss möglichst genau sein, er darf weder dehnbar noch auslegungsbedürftig sein. ${ }^{14}$ In der Realität jedoch lässt der Weisungsinhalt an Präzision häufig zu wünschen übrig, wenn es schlicht heisst: «Für die Dauer der Probezeit wird dem Beschuldigten die Weisung erteilt, sich einer psychologischen Behandlung im Sinne von Art. 94 StGB zu unterziehen.»15

9 Kontrolle: Im Kanton Zürich ist beispielsweise das Amt für Justizvollzug für die Bewährungshilfe und die Kontrolle der auferlegten Weisungen zuständig. 16 Die Weisungskontrolle ist dann besonders schwierig (oder faktisch unmöglich), wenn die betroffene Person nicht regelmässig in Kontakt mit der Behörde treten

ler Kommentar, Strafrecht I, Art. 1-11o StGB und Jugendstrafgesetz, 3. Auflage, Basel 2013 (fortan: BSK StGB-BearbeiterIn), N 17.

13 Zwingend im Urteilsdispositiv, siehe Urteil des Kantonsgerichts Luzern vom 23. Februar 1993, in: LGVE 1993 I Nr. 38; BSK StGB-Schneider/ Garré (Fn. 12), Art. 44 N 20; François Vouilloz, L'interdiction de stade et de périmètre. Règle de conduite en cas de sursis (art. 44 al. 2 CP et $24 \mathrm{~b}$ al. 1 LMSI), Jusletter vom 2. Juni 2008, Rz.10; Hans Schultz, Einführung in den Allgemeinen Teil des Strafrechts, Ein Grundriss, 2. Band, 4. Auflage, Bern 1982 (AT II), 112; derselbe, Der bedingte Strafvollzug nach dem Bundesgesetz vom 18. März 1971, ZStrR 1973, 52 ff., 61; Philipp Thormann/Alfred von Overbeck, Schweizerisches Strafgesetzbuch, Allgemeiner Teil, Zürich 1940, Art. $41 \mathrm{~N} 14$.

14 BSK StGB-Schneider/Garré (Fn. 12), Art. 44 N 29; vgl. auch BGE 105 IV 203 E. 2.b.

15 Urteil des Obergerichts Zürich SB150166 vom 13. Oktober 2015, Dispositivziff. 5, die vorinstanzliche Weisung bestätigend; ferner Urteil des Kantonsgerichts Freiburg 501201592 vom 6. Juni 2016, Dispositivziff. 8 Abs. 4; vgl. für weitere Beispiele Martino Imperatori, Weisungen, in: Benjamin Brägger (Hrsg.), Das schweizerische Vollzugslexikon, Basel 2014, 529 ff., 534 .

16 § 5 Justizvollzugsverordnung des Kantons Zürich (LS 331.1). 
muss, da sie - zu Recht - nicht unter dauernder Überwachung steht. ${ }^{17}$

Durchsetzung: Art. 95 Abs. 4 und 5 StGB sehen für den Fall der Missachtung der Weisung eine Reihe von Reaktionsmöglichkeiten vor: Verlängerung der Probezeit um höchstens die Hälfte der ausgesprochen Dauer, Änderung, Aufhebung einer Weisung oder Erteilung einer neuen. ${ }^{18}$ Die zwangsweise Durchsetzung der Weisung (bspw. die Zuführung zum Arzt) ist gesetzlich nicht vorgesehen und damit unzulässig. ${ }^{19}$ Als ultima ratio kann nach Abs. 5 die bedingte Strafe widerrufen werden, falls - und nur dann - sich die Rückfallprognose verschlechtert hat. Von dieser Möglichkeit wird in der Praxis jedoch sehr zurückhaltend Gebrauch gemacht. ${ }^{20}$ Im Regelfall bleibt es deshalb zunächst einmal bei einer Ermahnung. ${ }^{21}$ Seit 2015 wird mit Art. 295 StGB die Missachtung von Weisungen und Bewährungshilfe zusätzlich mit Busse bestraft, auch wenn keine Rückfallgefahr vorliegt. ${ }^{22}$

17 Vgl. hierzu Rudolf Schweizer, Die Rechtsstellung des mit bedingtem Strafvollzug Verurteilten im Sinne von Art. 41 Ziff. 3 StGB, ZStrR 58/1944, 348 ff., 351 ff.; vgl. auch BSK StGB-Impteratori (Fn. 12), Art. $93 \mathrm{~N} 20$; vgl. auch hinten IV.3.b)

18 Vgl. BSK StGB-Imperatori (Fn. 12), Art. 94 N 15.

19 So schon Alexander Lüthy, Der bedingte Strafvollzug im schweizerischen Recht, Diss. Bern 1948, 74 .

20 Vgl. BSK StGB-Imperatori (Fn. 12), Art. 95 N 16; so auch die schriftliche Auskunft des Amts für Justizvollzug des Kantons Zürich vom 31. Januar 2017.

21 So wohl auch Stefan Trechsel/Peter Abersold, Art. 95, in: Stefan Trechsel/Mark Pieth (Hrsg.), Schweizerisches Strafgesetzbuch, Praxiskommentar, 3. Auflage, Zürich/St. Gallen 2017 (fortan: PK3-BearbeiterIn) N 10.

22 Damit kam der Bundesrat einem Anliegen aus der Praxis nach, vgl. Botschaft vom 10. Oktober 2012 zur Volksinitiative «Pädophile sollen nicht mehr mit Kindern arbeiten dürfen» sowie zum Bundesgesetz über das Tätigkeitsverbot und das Kontakt- und Rayonverbot (Änderung des Straf-
11 Beendigung: Spätestens mit Ablauf der Probezeit (oder einer allenfalls kürzer festgelegten Dauer) ${ }^{23}$ fallen die erteilten Weisungen dahin. ${ }^{24}$ Eine Weisung kann aber auch dahinfallen, wenn sie unerfüllbar wird oder nicht mehr zweckmässig ist (vgl. Art. 95 Abs. 4 StGB), 25 oder ihr Zweck erreicht wurde. ${ }^{26}$

12 Weisungen werden nicht spezifisch erfasst. ${ }^{27}$ Das Bundesamt für Statistik weist für die Jahre 2014 und 2015 je ca. 1000 Anordnungen von «Betreuungen» aus, im Zuge derer entweder nur Weisungen oder Bewährungshilfe angeordnet oder beide kombiniert wurden. Davon wurden rund $15 \%$ zusammen mit einer (teil-) bedingten Strafe angeordnet. Diese verteilen sich ungefähr hälftig auf Geld- und Freiheitsstrafen. ${ }^{28}$

gesetzbuchs, des Militärstrafgesetzes und des Jugendstrafgesetzes) als indirektem Gegenvorschlag (BBl 2012 8819), 8867. Dies wurde bereits vorher teilweise so gehandhabt, indem einfach eine Strafe nach Art. 292 StGB bei Missachtung der Weisung angedroht wurde, so Urteil des Kantonsgerichts Graubünden SK1 1516 vom 5. Oktober 2015, Dispositivziff. 3 .

23 BSK StGB-Schneider/Garré (Fn. 12), Art. 44 N 19; PK3-Trechsel/Aebersold (Fn. 21), Art. 94 N 3; BGE 77 IV 71 E. 3; bestätigt in Urteil des Bundesgerichts 6S.79/2001 vom 26. Februar 2001, E. 2.c.

24 BSK StGB-Imperatori (Fn. 12), Art. 94 N 5; Christian Schwarzenegger/Markus Hug/Daniel Jositsch, Strafrecht II, Strafen und Massnahmen, Zürich/Basel/Genf 2007, 305.

25 Günter Stratenwerth, Schweizerisches Strafrecht, Allgemeiner Teil II: Strafen und Massnahmen, 2. Auflage, Bern 2006, § 5 N 82.

26 Schultz (Fn. 13, AT II), 112.

27 BSK StGB-Imperatori (Fn. 12), Art. 94 N 4.

28 Vgl. zum Ganzen Bundesamt für Statistik (BFS), Anordnungen von Bewährungshilfe und Weisungen, BFS-Nr. je-d-19.04.03.28, veröffentlicht am 29. August 2016. 


\section{Inhalt}

13 Der Inhalt der Weisung steht im Ermessen des Gerichts. ${ }^{29}$ Das Gericht soll sich dabei von fürsorgerischen, kriminalpädagogischen und medizinischtherapeutischen Bedürfnissen leiten lassen. ${ }^{30}$ Weisungen und deren Inhalt werden nicht systematisch erfasst, weshalb es kaum möglich ist, sich einen detaillierten Überblick über die Weisungspraxis zu verschaffen. ${ }^{31}$ Das Gesetz nennt die eingangs erwähnten fünf Arten von Weisungen (Art. 94 StGB). Diese würden als Vorschläge dienen, seien jedoch nicht abschliessend. ${ }^{2}$

Es erscheint grundsätzlich sinnvoll, die Weisungskompetenz offen zu gestalten, um eine Individualisierung der Sanktion zu ermöglichen. Allerdings schliesst dies $\mathrm{m}$. E. nicht aus, die möglichen Weisungsarten zumindest grob zu definieren, so wie das der Gesetzgeber mit der beispielhaften Aufzählung bereits ansatzweise getan hat. Weisungen, welche in keiner Weise von den gesetzlichen Beispielen umfasst werden oder mit diesen in $\mathrm{Zu}$ sammenhang stehen, liegen wohl jenseits einer zulässigen Dehnung des strafrechtlichen Legalitätsprinzips, da für den Einzelnen sonst nicht erkennbar wäre, welche Sanktionen ihm drohen.33 Insbeson-

BGE 130 IV 1 E. 2.1; Urteil des Bundesgerichts 6B_166/2016 vom 7. Juli 2016, E. 4.2; zustimmend BSK StGB-Schneider/Garré (Fn. 12), Art. $44 \mathrm{~N} 26$, «jede denkbare Weisung».

30 BGE 107 IV 88 E. 3.a.

${ }^{31}$ BSK StGB-Imperatori (Fn. 12), Art. $94 \mathrm{~N} 4$.

32 So die h. L., BSK StGB-Imperatori (Fn. 12), Art. 94 N 19; PK3-Trechsel/Aebersold (Fn. 21), Art. $94 \mathrm{~N} 2$, unklar jedoch $\mathrm{N}$ 3; Stratenwerth (Fn. 25, AT II), § $5 \mathrm{~N} 74$.

33 Ausführlich Fellmann (Fn. 50), 73 ff. und $77 \mathrm{ff}$.; so auch Hans-Jürgen Bruns, Rechtsgrundlage und Zulässigkeitsgrenzen strafrichterlicher Auflagen und Weisungen, Ungültigkeit der sozialtherapeutischen Generalklausel des StGB?, GA 1959, dere weil es an gesetzlichen Leitlinien zur Weisungsfindung fehlt.34 Darüber hinaus hätten mindestens die zulässigen Weisungsarten abschliessend geregelt werden können und auch sollen.35 So wäre Art. 94 StGB bspw. um den Zusatz «Kontakt» zu ergänzen, wollte man diese Weisungen beibehalten.

15 Die Betrachtung beschränkt sich folgend auf drei Weisungsarten: Berufsausübung, Kontakt und ärztliche bzw. psychologische Betreuung. 36

16 Weisungen betreffend die Berufsausübung können verschieden ausgestaltet sein. So konnte es sich dabei bis anhin um ein Verbot handeln, einen bestimmten Beruf auszuüben,37 diesen zu wechseln, $3^{8}$ oder eine selbständige Erwerbstätigkeit auszuüben. 39 Fraglich ist, ob dies nach der Änderung der Massnahme des Berufsverbots zum seit Juni 2015 neu geltenden Tätigkeitsverbot (Art. $67 \mathrm{StGB}$ ) immer noch sinnvoll ist bzw. auch davor schon sinnvoll war.40 Lehner fragt sich bspw. zu Recht, inwiefern ein Berufsver-

193 ff., 207; Bericht VE-StGB/1993 (Fn. 8), 15, wonach der Begriff «Sanktion» neben Strafen und Massnahmen alle vom Strafgesetz vorgesehenen Rechtsfolgen umfasse; ebenso Botschaft, BBl 1999 II 1979 (Fn. 9), 1991.

34 Fellmann (Fn. 50), 73; BSK StGB-Imperatori (Fn. 12), Art. $94 \mathrm{~N} 6 \mathrm{f}$.

35 So auch einzelne Stimmen in der Beratung der Expertenkommission, vgl. Protokolle, Expertenkommission (Fn. 6), 441; vgl. auch Imperatori (Fn. 15, Vollzugslexikon), $530 \mathrm{f}$.

36 Für einen detaillierten Überblick über die Weisungspraxis vgl. BSK StGB-Imperatori (Fn. 12), Art. $94 \mathrm{~N} 1 \mathrm{ff}$;; ferner auch Imperatori (Fn. 15, Vollzugslexikon), $531 \mathrm{ff}$.

37 BSK StGB-Imperatori (Fn. 12), Art. 94 N 12; BGE 105 IV 289 f., hier spezifisch der Handel mit Sexartikeln.

38 BGE 106 IV 325 E. 2a; BSK StGB-Imperatori (Fn. 12), Art. 94 N 12.

39 Vgl. BSK StGB-Imperatori (Fn. 12), Art. 94 N 12, mit weiteren Beispielen.

40 Dazu hinten IV.2. 
bot überhaupt zur Resozialisierung beitragen soll, wenn es diese nicht sogar behindert. ${ }^{41}$ Daneben werden auch Weisungen erteilt, welche die Berufsausübung zwar betreffen, aber nicht im Sinne eines Verbots einschränken, wie bspw. das Gebot, der Bewährungshilfe Einsicht in die Geschäftskorrespondenz zu gewähren. 42

17 Unter Weisungen betreffend den Aufenthalt fielen bisher Weisungen wie bspw. das Verbot, gewisse Orte aufzusuchen (Rayonverbot)43, den Wohnort nicht ohne Rücksprache mit der Bewährungshilfe zu wechseln oder auch an einem bestimmten Ort Wohnsitz zu nehmen. 44

M. E. nicht vom Wortlaut gedeckt, aber teilweise ebenfalls unter "Aufenthalt» subsumiert wird das Kontaktverbot, 45 was das Obergericht Zürich erst kürzlich bestätigt hat. 46

Mit einer Weisung bezüglich ärztlicher oder psychologischer Betreuung sollen nach dem Bundesrat nur bestimmte, nicht allzu tief in die Freiheitsrechte des Betroffenen eingreifende Therapien ver-

41 Marco Lehner, Das Berufsverbot als Sanktion im schweizerischen Strafrecht, Diss. Zürich 1990, 1991, 86, relativierend jedoch $88 \mathrm{f}$.

42 BGE 107 IV 88 E. 3.b; ferner BSK StGBImperatori (Fn. 12), Art. 94 N 12.

43 So bspw. ein Stadionverbot sowie ein Rayonverbot im Umkreis von 400 Metern des Stadions an Spieltagen für die Dauer der Probezeit von drei Jahren, Urteil des Kantonsgerichts Wallis vom 29. Dezember 2006, in: ZWR 2007, 326 ff.; für das Verhältnis zu Stadion- und Rayonverboten nach BWIS siehe Vouilloz (Fn. 13), Rz. 6 ff.

44 Vgl. BSK StGB-Imperatori (Fn. 12), Art. 94 $\mathrm{N} 13 \mathrm{f}$.

45 Vgl. BSK StGB-Schneider/Garré (Fn. 12), Art. 44 $\mathrm{N} 40$.

46 Urteil des Obergerichts Zürich SB150260 vom 2. Oktober 2015, Dispositivziff. 4, allerdings vor Inkrafttreten des Kontaktverbots als Massnahme, siehe Ziff. 2.1.1 ff. fügt werden; ausdrücklich ausgenommen sei die Verwahrung in einer Anstalt,47 was selbstverständlich erscheint. Gemeint sind also sowohl Therapien in ambulanter Form als auch Interventionen geringerer Intensität wie bspw. die regelmässige Urinkontrolle, um den Verzicht auf Drogenkonsum zu überprüfen. 48 Auch kommt es in der Praxis vor, dass der Verurteilte angewiesen wird, eine freiwillig aufgenommene Therapie fortzuführen. 49

\section{Voraussetzungen}

2o Die offene Ausgestaltung der Weisungskompetenz in Art. 94 StGB wird von einem Teil der Lehre kritisiert, 50 von einem anderen Teil sowie vom Gesetzgeber hingegen für ausreichend konkret oder sogar notwendig erachtet. $5^{1}$

21 Bedauerlich ist in diesem Zusammenhang auch, dass der Gesetzgeber weder

47 So Botschaft, BBl 1999 II 1979 (Fn. 9), 2068, damit wohl stationäre Massnahmen meinend, egal welcher Art.

48 BSK StGB-Schneider/Garré (Fn. 12), Art. 44 $\mathrm{N} 44$.

49 BSK StGB-Schneider/Garré (Fn. 12), Art. 44 $\mathrm{N} 44$; in Urteil des Bundesgerichts 6S.148/2004 vom 28. Juli 2004, zumindest beantragt; kritisch Monique Gisel-Bugnion, Punir sans prison, Quelques suggestion, Genf 1984, $45 \mathrm{f}$.

50 Vgl. BSK StGB-Imperatori (Fn. 12), Art. 94 N 6 f., findet dies «bedauerlich» und kritisiert die «armselige Regelungsdichte»; siehe auch Stratenwerth (Fn. 25, AT II), § 5 N 74, der schreibt, eine Abgrenzung allein aus Zweckbestimmungen und rechtsstaatlichen Grundsätzen sei «relativ unbestimmt»; ferner Brigitta Fellmann, Die Weisung gemäss Art. 41 Ziff. 2 StGB, unter besonderer Berücksichtigung der verfassungsrechtlichen Problematik, Diss. Basel 1973, 73.

51 Botschaft, BBl 1999 II 1979 (Fn. 9), 2127; Schwarzenegger/Hug/Jositsch (Fn. 24), $301 \mathrm{ff}$; Michel Dupuis/Bernhard Geller/Gilles Monnier/Laurent Moreillon/Christophe Piguet/ Christian Bettex/Daniel Stoll (Hrsg.), Petit Commentaire, Code pénal, Basel 2012, Art. 94 N 2; unklar PK3-Trechsel/Aebersold (Fn. 21), Art. 94. 
spezifische Weisungsvoraussetzungen normiert noch den spezialpräventiven oder resozialisierenden Zweck im Gesetz festgehalten hat. ${ }^{2}$

Einigkeit besteht zumindest darüber, dass aufgrund der offenen Formulierung der Bestimmung eine Begrenzung durch eine gewissenhafte Anwendung des Verhältnismässigkeitsprinzips unumgänglich ist.53

Weitere Voraussetzungen können nur hergeleitet werden, was folgend versucht wird.

\section{a) Koppelung an bedingte Strafe}

Weisungen sind nur zusammen mit einer bedingten Strafe möglich (Art. 44 Abs. 2 StGB). Dies bedeutet, dass eine Weisung - im Gegensatz zu einer Massnahme nur nach einer schuldhaft begangenen Tat erteilt werden kann. ${ }^{54}$ Eine Kombination mit einer unbedingten Strafe ist nicht möglich. Damit sind die Voraussetzungen einer bedingten Strafe indirekt auch Weisungsvoraussetzungen.

Der bedingte Strafvollzug hat zum einen eine formelle Voraussetzung, nämlich die Art der Sanktion.55 Voll aufgeschoben werden können nur Geldstrafen bis und mit 18o Tagessätzen und Freiheitsstrafen von bis zu zwei Jahren (Art. 42 Abs. 1 StGB). Allerdings ist darüber hinaus ein teilweiser Aufschub für Freiheitsstrafen

52 Vgl. Imperatori (Fn. 15, Vollzugslexikon), 530.

53 BSK StGB-Imperatori (Fn. 12), Art. 94 N 7; Stratenwerth (Fn. 25, AT II), § $5 \mathrm{~N} 73 \mathrm{f}$.

54 Beschränkt auf Weisungen nach Art. 44 Abs. 2 StGB; vgl. Botschaft, BBl 1999 II 1979 (Fn. 9), 2068.

55 Stratenwerth (Fn. 25, AT II), § 5 N 13; BSK StGB-Schneider/Garré (Fn. 12), Art. 42 N 1.

von bis zu drei Jahren möglich, welcher auch die Weisungserteilung erlaubt.

26 gewährt, wenn ein unbedingter Vollzug zur Vermeidung einer erneuten Deliktsbegehung nicht notwendig erscheint, mithin also keine schlechte Prognose gestellt wird (materielle Voraussetzung). 56

27 Sinnvollerweise muss das Gericht bei der Prognosestellung die Möglichkeiten der Weisung bereits miteinbeziehen.57 Wird einem Beschuldigten vorerst eine negative Prognose gestellt, so muss in einem zweiten Schritt geprüft werden, ob ihm unter Berücksichtigung allfälliger Weisungen nicht doch eine bessere Prognose gestellt und damit der bedingte Vollzug gewährt werden kann. Weisungen müssen m. E. prognoserelevant sein. $5^{8}$ Demjenigen, der die Voraussetzungen des bedingten Strafvollzuges ohne begleitende Anordnungen erfüllt, dürfen keine Weisungen erteilt werden, da diese dann nicht erforderlich sind. 59

Dass diese Prognoseschwelle keinen genau bestimmbaren Wert darstellt, ist klar. ${ }^{60}$ Auch vertreten die h. L. und das

56 BSK StGB-Schneider/Garré (Fn. 12), Art. 42 N 38; BGE 135 IV 180 E. 2.1.

57 Gängige Praxis seit BGE 99 IV 68; PK3Trechsel/Pieth (Fn. 21), Art. $42 \mathrm{~N}$ 14; ebenso Fellmann (Fn. 50), 15 f.; Botschaft, BBl 1999 II 1979 (Fn. 9), 2054; BSK StGB-Schneider/Garré (Fn. 12), Art. 42 N 84; Rottenberg (Fn. 64), 66 f.; Thormann/von Overbeck (Fn. 13), Art. 41 N 13; anders noch BGE 83 IV 65.

58 So im Ergebnis auch Urteil des Bundesgerichts 6B_652/2016 vom 28. März 2017, E. 3.3.2., «im Hinblick auf eine günstige Legalprognose».

59 Vgl. vorne Rz. 35; MüKo-Gross (Fn. 71), § 56c N 5, "'bedarf' der Verurteilte keiner Weisung"; vgl. auch Fellmann (Fn. 50), 68.

60 Vgl. Stratenwerth (Fn. 25, AT II), § $5 \mathrm{~N}$ 34; «intuitive Methode» PK 3 -Trechsel/Pieth (Fn. 21), Art. 42 N 9. 
Bundesgericht in Übereinstimmung mit dem Wortlaut von Art. 42 Abs. 1 StGB die Meinung, dass heute im Zweifel für den bedingten Vollzug zu entscheiden ist, also eine gesetzliche Vermutung zu dessen Gunsten besteht. ${ }^{61}$ Im Ergebnis heisst dies aber auch, dass man sich eher für eine Weisung entscheiden muss als für den unbedingten Vollzug, was zumindest bei Freiheitsstrafen nicht zu kritisieren ist.

\section{b) Öffentliches Interesse und Zweckbindung}

Was nicht vom öffentlichen Interesse umfasst ist, darf nicht Zweck der Weisung sein. ${ }^{62}$ Das zentrale öffentliche Interesse ist die Verhütung weiterer Straftaten der beschuldigten Person (Spezialprävention). ${ }^{63}$ Allerdings muss aufgrund der Koppelung an die bedingte Strafe auch der bedingte Strafvollzug miteinbezogen werden, dessen Zweck bspw. auch die Vermeidung schädlicher Freiheitsstrafen umfasst. ${ }^{64}$ Daraus ergibt sich die enge Verbindung von Spezialpräventions- und Resozialisierungsgedanken. ${ }^{65}$ Mit erzieherischen Mitteln wird versucht, die Bewährungschancen des Verurteilten $\mathrm{zu}$ verbessern, ${ }^{66}$ also insbesondere die

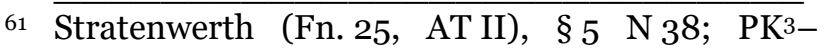
Trechsel/Pieth (Fn. 21), Art. 42 N 8; BSK StGBSchneider/Garré (Fn. 12), Art. 42 N 34; BGE 135 IV 180 E. 2.1.

62 Vgl. Astrid Epiney, Art. 36, in: Bernhard Waldmann/Eva Maria Belser/Astrid Epiney (Hrsg.), Basler Kommentar, Schweizerische Bundesverfassung (BV), Basel 2015 (fortan BSK BVBearbeiterIn), N 55.

63 Vgl. für Massnahmen Stratenwerth (Fn. 25, AT II), \& $8 \mathrm{~N} 3$.

64 Vgl. bspw. Schultz (Fn. 13, AT II), 111; Vera Rottenberg, Der bedingte Strafvollzug, Diss. Zürich 1972, Zürich 1973, 1, 3 f.

65 Vgl. BSK StGB-Schneider/Garré (Fn. 12), Vor Art. $42 \mathrm{~N} 6$.

66 Früher sah das Bundesgericht noch eine innerliche Besserung vor, vgl. BGE 94 IV 11 E. 1. Eine
Rückfallgefahr zu senken. ${ }^{67}$ Damit wirkt die Weisung in die gleiche Richtung wie die meisten Massnahmen - ersterer kommt aber kein Sicherungszweck zu. ${ }^{68}$

30 Das Bundesgericht hält sodann ohne Begründung fest, Weisungen müssten in erster Linie im Interesse des Verurteilten liegen. ${ }^{69}$ Dem Bundesgericht ging es wohl darum, zu verdeutlichen, dass Weisungen nicht primär dem Schutz Dritter dienen oder überwiegend punitive oder generalpräventive Zwecke verfolgen dürfen. ${ }^{70}$ Rückschauend hätte das Bundesgericht allerdings besser auf dieses Lippenbekenntnis verzichtet, weil das Argument ad absurdum geführt werden kann und auch tatsächlich wird. So wird bspw. schlicht behauptet, eine Weisung, sich

Veränderung der inneren Gesinnung kann aber in einem modernen Rechtsstaat nicht mehr Ziel des bedingten Strafvollzuges oder der Weisung sein. Vgl. dazu statt vieler Claus Roxin, Strafrecht Allgemeiner Teil, Band 1, Grundlagen - der Aufbau der Verbrechenslehre, 4. Auflage, München 2006, § 3 N 17; ebenso Stratenwerth (Fn. 25, AT II), \& $8 \mathrm{~N} 5$.

67 Vgl. BGE 137 IV 72 E. 2.4; vgl. auch BSK StGBImperatori (Fn. 12), Art. 94 N 9; Stratenwerth (Fn. 25, AT II), § 5 N 72; Weisungen haben «prophylaktisch zu wirken und die Gefahr des Rückfalls zu vermindern», so Thormann/von Overbeck (Fn. 13), Art. $38 \mathrm{~N} 12$.

68 Vgl. Botschaft, BBl 1999 II 1979 (Fn. 9), 2068.

69 BGE 106 IV 325 E. 1; BGE 130 IV 1 E. 2.1 «elle doit être conçue en premier lieu dans l'intérêt du condamné»; aufgenommen in der Botschaft, BBl 1999 II 1979 (Fn. 9), 2128; vgl. PK3Trechsel/Aebersold (Fn. 21), Art. 94 N 3; Günter Stratenwerth/Wolfgang Wohlers, Schweizerisches Strafgesetzbuch, Handkommentar, 3. Auflage, Bern 2013, Art. 94 N 1; Daniel Mühlemann, Prävention von Wirtschaftsdelikten durch Berufsverbote, Diss. Zürich 1987, 182, «grosszügige Auslegung des Begriffs der 'Interessen des Verurteilten'».

70 Siehe dazu PK3-Trechsel/Aebersold (Fn. 21), Art. 94 N 3; Stratenwerth (Fn. 25, AT II), § 5 N 72; BSK StGB-Imperatori (Fn. 12), Art. 94 N 9; BGE 94 IV 11 E. 1; BGE 105 IV 289 f.; auch wenn sie für den Verurteilten durchaus pönalen Charakter haben kann, so in BGE 108 IV 152 E. 3.a f. 
dem sexuell missbrauchten Opfer nicht mehr zu nähern, läge im Interesse des Täters. ${ }^{71}$ Dieser Gedanke erscheint jedoch zirkulär, läge nach dieser Argumentation doch jede das Rückfallrisiko verringernde Intervention im Interesse des Betroffenen.

Teilweise wird versucht, ein objektiviertes «wohlverstandenes» Interesse der beschuldigten Person festzustellen.72 Was dieses genau sein soll, ist nicht leicht zu beantworten.73 Dieses Konstrukt repräsentiert jedenfalls kein privates Interesse. Dass mit dem bedingten Strafvollzug das erreicht werden soll, was die Gesellschaft für den Täter für das Beste hält, nämlich Resozialisierung und Rückfallverminderung, ist offensichtlich. Dies stellt den Zweck der Weisungen und damit primär ein öffentliches Interesse dar. Ein darüber hinausgehendes Interesse des Betroffenen muss gerade nicht bestehen. Weisungen müssen sinnvollerweise auch dann zulässig sein, wenn sie nicht primär im Interesse der beschul-

So BSK StGB-Schneider/Garré (Fn. 12), Art. 44 N 28; vgl. auch Vouilloz (Fn. 13), Rz. 28, "L'interdiction judiciaire de stade et de périmètre vise principalement à protéger le hooligan contre la pratique du hooliganisme. »; zustimmend auch Sven Zimmerlin, Uferloses Strafrecht? Von unnötigen und unklaren Bestimmungen - und solchen, die noch umzusetzen sind, forumpoenale 4/2013, 228 ff., 231; vgl. dazu auch Gross, § 56c, in: Joecks Wolfgang/Miebach Klaus (Hrsg.), Münchener Kommentar zum Strafgesetzbuch: StGB, Band 2, §§ 38-79b, München 2016 (fortan: MüKo-BearbeiterIn), N 27; vgl. auch Gisel-Bugnion (Fn. 49), 85 f.

72 So ausnahmsweise BGE 103 IV 134 E. 2, sowie BGE 137 IV 72, E. 2.4; BSK StGB-Imperatori (Fn. 12), Art. 94 N 9; Peter Mösch Payot, Anordnung von Pflichtberatung und Lernprogrammen im Rahmen von strafrechtlichen Sanktionen, insb. als Weisungen, Jusletter vom 4. Juni 2012, Rz. 18; Mühlemann (Fn. 69), 177, 182.

73 Vgl. ausführlich zu Massnahmen allgemein, Stratenwerth (Fn. 25, AT II), § $8 \mathrm{~N} 27 \mathrm{ff}$. digten Person liegen. ${ }^{74}$ In solchen Fällen sollte dies schlicht zugegeben werden, anstatt die Konformität mit den Interessen des Betroffenen zu behaupten.75

32 Es ist grundsätzlich richtig, dass der verfolgte spezialpräventive Zweck nicht zwingenderweise im Widerspruch mit den Interessen des Betroffenen steht. 76 Dies kann bei der Interessenabwägung insofern berücksichtigt werden, als es dem Eingriff an Gewicht nimmt, wenn dieser dem Interesse des Betroffenen entspricht. 77

\section{c) Verhältnismässigkeit}

33

Dem Verhältnismässigkeitsprinzip kommt bei der Bestimmung der Grenzen von Weisungen eine zentrale Bedeutung zu. Die Verhältnismässigkeitsprüfung umfasst anerkanntermassen drei Teilgehalte: die Eignung, die Erforderlichkeit und die Zumutbarkeit bzw. Verhältnismässigkeit i.e.S., welche eine Abwägung

74 Im Ergebnis ebenso Mühlemann (Fn. 69), 177 f.; vgl. auch Peter Häfliger, Revalorisation de la probation, Nouvelles bases légales pour la probation dans le CP révisé, info bulletin 2/2008, BJ, Informations sur l'exécu-tion des peines et mesures, $4 \mathrm{ff} ., 4$, der in Bezug auf die Bewährungshilfe ausführt, dass die Idee der Beschränkung auf die Interessen der beschuldigten Person bereits nach dem Vernehmlassungsverfahren verworfen wurde.

75 Bspw. wird mit einem Rayon- und Kontaktverbot für den gewalttätigen Ehemann das öffentliche spezialpräventive Interesse ausgedrückt, Gewaltakte gegenüber der Ehefrau möglichst zu vermeiden, während das faktische Interesse des Ehemanns allenfalls auf einen Versöhnungsversuch abzielt.

76 So BSK StGB-Schneider/Garré (Fn. 12), Art. 44 $\mathrm{N}$ 27; übernommen in Urteil des Obergerichts Zürich SB150260 vom 2. Oktober 2015, Ziff. 2.2.1.

77 Stratenwerth (Fn. 25, AT II), § 8 N 27. 
zwischen den betroffenen öffentlichen und privaten Interessen darstellt. 78

Die Eignung findet ihre Grenze im Zweck, welcher Weisungen zugrunde liegt.79 Eine Weisung, die sich nicht eignet, den spezialpräventiven Zweck zu erreichen, darf nicht erteilt werden. ${ }^{80}$ In die gleiche Kategorie fallen unerfüllbare Weisungen. ${ }^{81}$ Die Weisung muss ausserdem in einem Zusammenhang mit der Tat stehen, da sich ansonsten kaum ein die Rückfallwahrscheinlichkeit verringernder Effekt einstellt. ${ }^{82}$

Die Weisung muss weiter erforderlich sein, das heisst, sie muss das mildeste geeignete Mittel sein. ${ }^{83}$ Ist nur ein einziger staatlicher Eingriff geeignet, dann entfällt die Prüfung der Erforderlichkeit. ${ }^{84}$ Beim bedingten Strafvollzug stellt bereits die Verurteilung einen staatlichen

Vgl. Benjamin Schindler, Art. 5, in: Bernhard Ehrenzeller/Benjamin Schindler/Rainer J. Schweizer/Klaus A. Vallender (Hrsg.), St. Galler Kommentar, Die Schweizerische Bundesverfassung, 3. Auflage, Zürich/Basel/Genf 2014 (fortan: SGK BV-BearbeiterIn), N 48; SGK BVSchweizer, Art. $36 \mathrm{~N} 37 \mathrm{ff}$.

79 Vgl. Walter Stree/Jörg Kinzig, § 56c, in Adolf Schönke/Horst Schröder/Albin Eser (Hrsg.), Strafgesetzbuch Kommentar, 29. Auflage, München 2014 (fortan: Schönke/SchröderBearbeiterIn), N 6; allgemein Ulrich Häfelin/Georg Müller/Felix Uhlmann, Allgemeines Verwaltungsrecht, 7. Auflage, Zürich/St. Gallen 2016, Rn. 522.

80 Vgl. Gisel-Bugnion (Fn. 49), 87, welche u. a. aus diesem Grund für eine Abschaffung der Weisungen plädiert, da sie generell an deren Eignung zur Prävention zweifelt.

81 BGE 94 IV 11 E. 1.

82 Vgl. Stratenwerth (Fn. 25, AT II), § 5 N 72; BGE 102 IV 8 E. 1. Dies wohl nach derselben Logik des Erfordernisses des Deliktskonnexes bei Massnahmen, siehe bspw. BSK StGB-Heer (Fn. 12), Art. $56 \mathrm{~N} 47$.

83 BSK BV-Epiney (Fn. 62), Art. 36 N 56; SGK BVSchweizer (Fn. 78), Art. 36 N 39; BGE 140 I 2 E. 9.2.2; Häfelin/Müller/Uhlmann (Fn. 79), Rn. 530.

84 Häfelin/Müller/Uhlmann (Fn. 79), Rn. 527.
Eingriff dar, ${ }^{85}$ da sie auch alleine geeignet sein kann, den Täter von weiteren Straftaten abzuhalten. Das bedeutet, dass bei Personen, die keiner Resozialisierung bedürfen bzw. eine genügend gute Prognose gestellt bekommen, keine Weisungen zulässig sind. Aus dem Kriterium der zeitlichen Erforderlichkeit ergibt sich zudem, dass Weisungen auch für eine kürzere Dauer als die Probezeit angeordnet werden können, wenn dies ausreicht. 86

36 Grundsätzlich muss das öffentliche Interesse an der Weisung die dadurch beeinträchtigten privaten Interessen überwiegen. ${ }^{87}$ Das Bundesgericht führt dazu aus, dass Weisungen nur dann erteilt werden dürfen, «wenn der Eingriff in die Persönlichkeitsrechte des Täters aufgewogen wird durch die Art und die Schwere der zu sühnenden Tat oder der Delikte, die der Täter in Zukunft allenfalls begehen könnte, oder durch die Grösse der Wahrscheinlichkeit neuer Straftaten.»88

Unklar ist, weshalb hier die Art und die Schwere des begangenen Deliktes berücksichtigt werden sollen. Dies ist abzulehnen, da sich diese bereits im Strafrahmen widerspiegeln und nichts über zukünftiges Verhalten aussagen. ${ }^{89}$

Schneider/Garré gehen sogar so weit, zu behaupten, dass die Weisung umso ein-

85 Vgl. hinten III.2.

86 So verstanden BSK StGB-Schneider/Garré (Fn. 12), Art. 44 N 19; BGE 77 IV 71 E. 3; bestätigt in Urteil des Bundesgerichts 6S.79/2001 vom 26. Februar 2001, E. 2.c; vgl. allgemein Häfelin/Müller/Uhlmann (Fn. 79), Rn. 543.

87 Häfelin/Müller/Uhlmann (Fn. 79), Rn. 556 f.

88 BGE 107 IV 88 E. 3.a, mit Verweis auf Markus Zingg, Der Ausbau der Schutzaufsicht für Straffällige zum Sozialdienst der Justizdirektion des Kantons Zürich, Diss. Zürich 1976, $31 \mathrm{ff}$.

89 So auch Imperatori (Fn. 15, Vollzugslexikon), 531. 
schneidender sein dürfe, je höher der Wert des verletzten Rechtsgutes sei.90 Dies ist ebenso abzulehnen, da der Wert des Rechtsguts bereits bei der Festlegung des abstrakten Strafrahmens durch den Gesetzgeber berücksichtigt wurde und er sich damit ebenfalls in der ausgefällten Strafe widerspiegelt.91

\section{Rechtfertigung der Weisung}

39 Als Rechtfertigungen für Weisungen wird oft lapidar festgestellt, dass der Verurteilte ja der unbedingten Strafe entgeht, er deshalb einzelne Eingriffe hinzunehmen hat.92 $\mathrm{Ob}$ dem so ist, soll nachfolgend geklärt werden.

\section{Charakter der Weisung}

40 Grundsätzlich sollten Weisungen ausschliesslich spezialpräventive Zwecke verfolgen, ein Strafcharakter muss ihnen gerade fehlen.93 Allerdings wird im schweizerischen Recht nicht wie im deutschen Recht zwischen pönalen Auflagen und nicht-pönalen Weisungen unterschieden.94 Dies führt dazu, dass Gerichte bei einzelnen Weisungen (bspw. bezüglich Schadensdeckung oder Zahlung von Unterhaltsbeiträgen95) erhebliche

90 BSK StGB-Schneider/Garré (Fn. 12), Art. 44 N 30; auch Schultz (Fn. 13, AT II), 111.

91 Vgl. Gerhard Fiolka, Das Rechtsgut als Massstab des Verschuldens in Art. 47 StGB, ZStrR 125/2007, 133 ff., 150 .

92 Vgl. bspw. BGE 137 IV 72 E. 2.4.

93 Vgl. Botschaft, BBl 1999 II 1979 (Fn. 9), 2128 f.

94 Vgl. Schönke/Schröder-Stree/Kinzig (Fn. 79), $\S 56 \mathrm{c}$ N 1 f.; MüKo-Gross (Fn. 71), § 56b N 2; Bernd-Dieter Meier, Strafrechtliche Sanktionen, 4. Auflage, Heidelberg 2015, 120 ff.; Rottenberg (Fn. 64), 114 ff.; Auflagen erwähnt und für das Schweizer Recht verworfen in Hans Schultz, Bericht zur Revision des Allgemeinen Teil und des Dritten Buches «Einführung und Anwendung des Gesetzes» des Schweizerischen Strafgesetzbuches, Bern 1987, 143.

95 Stratenwerth (Fn. 25, AT II), § 5 N 83.
Schwierigkeiten haben, einen teilweise pönalen Zweck (Schuldausgleich) wegzuargumentieren. ${ }^{96}$ Lässt man diese Weisungen jedoch ausser Acht, muss ein unverkennbarer Massnahmencharakter der Weisung anerkannt werden. 97

41 Zur Rechtfertigung von Weisungen müssten deshalb wohl am ehesten die für die Massnahmen entwickelten Grundsätze herangezogen werden. Da Massnahmen - anders als Strafen - gerade nicht durch das Schuldprinzip begrenzt sind, bedarf es anderer begrenzender Prinzipien. ${ }^{8}$ Es steht die Sozialgefährlichkeit des Täters im Mittelpunkt.99 Die Massnahme soll das Rückfallrisiko verringern, indem sie die individuellen Faktoren verbessert, welche zur Delinquenz geführt haben, ${ }^{100}$ oder auf die Tatgelegenheitssituation einwirken (Tätigkeits-, Kontakt-, und Rayonverbot).

42 Eine Rechtfertigung für den Eingriff kann sich in diesen Situationen nur aus einer Güterabwägung ergeben. Gegeneinander abgewogen werden die Schwere und Wahrscheinlichkeit der zu erwartenden weiteren Tat und die Schwere der Einschränkung, die der Verursacher der Gefahr zu tragen hätte. ${ }^{101}$ Da die Weisung jedoch im Zusammenhang mit dem be-

$9 6 \longdiv { \text { Vgl. BGE } 1 2 7 \text { IV 145, E. 2.c.bb; kritisch auch Stra- } }$ tenwerth (Fn. 25, AT II), § 5 N 78; $\mathrm{PK}^{3-}$ Trechsel/Aebersold (Fn. 21), Art. 94 N 7.

97 Ebenfalls für Massnahmencharakter Zingg (Fn. 88), 29; vgl. auch Gisel-Bugnion (Fn. 49), 83, welche feststellt, dass diese Frage kaum untersucht wurde.

98 Roxin (Fn. 66), § 3 N 65 ff.

99 BSK StGB-Heer (Fn. 12), Vor Art. 56 N 2.

100 BGE 137 IV 201 E. 3.1: «Au contraire de l'internement, qui consiste principalement à neutraliser l'auteur, la mesure thérapeutique institutionnelle cherche à réduire le risque de récidive par une amélioration des facteurs inhérents à l'intéressé.»

101 So bei Roxin (Fn. 66), § 3 N 66. 
dingten Strafvollzug erteilt wird, muss sowohl die Schwere als auch die Wahrscheinlichkeit der zu erwartenden Rückfälle gering ausfallen, womit sich daraus i. d. R. keine eigenständige Rechtfertigung ableiten lassen wird.

Gerade weil Weisungen vom Gericht nur zusammen mit dem bedingten Strafvollzug erteilt werden, kann sich eine Rechtfertigung wohl auch nicht getrennt davon ergeben.

\section{Charakter der bedingten Strafe}

44 Zum Charakter der bedingten Strafe gibt es zahlreiche Lehrmeinungen. Sie lassen sich grob in drei Gruppen einteilen: diejenigen, welche vom vorwiegenden Straf(1) oder Massnahmencharakter (2) ausgehen und diejenigen, welche sich für eine Art «Mischcharakter» oder einen sogar gänzlich eigenen Charakter aussprechen (3). ${ }^{102}$

Die h. L. geht heute vom Strafcharakter der bedingten Strafe aus. ${ }^{103}$ Eine Strafe wird aufgeschoben und macht einer Probezeit Platz. Bei dieser Probezeit, während der ein Verurteilter unter ständiger Drohung des Strafwiderrufs leben muss, handelt es sich um eine Strafe, ${ }^{104}$ selbst

102 Siehe dazu statt vieler Lüthy (Fn. 19), 14 ff.; vgl. Schultz (Fn. 13, AT II), 97 f.;

103 Vgl. BSK StGB-Schneider/Garré (Fn. 12), Vor Art. 42 N 52; Mark Pieth, Bedingte Freiheit, Disziplinierung zwischen Gnade und Kontrolle, Habil. Basel 1989, Basel/Genf/München 2001, 268, spricht von einem «Damoklesschwert»; Stratenwerth (Fn. 25, AT II), § 5 N 8; Ernst Hafter, Lehrbuch des schweizerischen Strafrechts, Allgemeiner Teil, 2. Auflage, Bern 1946, 327, von einer «Strafnatur»; vgl. auch die Zusammenstellung der Lehrmeinungen bei Widmer, $15 \mathrm{ff}$.

104 Vgl. Sandro Cimichella, Die Geldstrafe im Schweizer Strafrecht, unter Berücksichtigung der Problematik zum bedingten Vollzug, Diss. Zürich 2005, Bern 2006, 213; Pieth (Fn. 103), 221, 268. wenn keine Weisungen erteilt werden. Auch das mit einer Verurteilung verbundene Stigma sowie der Strafregistereintrag sind Strafbestandteile. ${ }^{105}$

46 Der bedingte Vollzug stellt - bei gleicher Eignung, die Strafzwecke zu erreichen das mildere Mittel dar. ${ }^{106}$ Man kann wohl so weit gehen, zu behaupten, dass der bedingte Strafvollzug mit seinen Individualisierungsmöglichkeiten häufig sogar das besser geeignete Mittel darstellen kann. ${ }^{107}$

47 Das Schuldprinzip soll die Strafe nach oben, nicht jedoch nach unten begrenzen. ${ }^{108}$ Ein «Untermassverbot», wie es bei therapeutischen Massnahmen angenommen wird, besteht bei bedingten Strafen gerade nicht. ${ }^{109}$ Die schuldadäquate Strafe wurde festgestellt, sie wird aber nicht vollzogen und damit in analogem quasi nicht ausgeschöpft. Dies kann aber nur gelten, solange die Strafe nicht doch vollzogen wird.

48 Die Gewährung des bedingten Vollzuges ist damit eine Modifikation der schuldadäquaten Strafe, ${ }^{110}$ welche deren Individualisierung erlaubt, sie muss aber nicht zwingend milder sein. ${ }^{111}$

105 Pieth (Fn. 103), 421, sieht darin sogar den Kern des bedingten Strafvollzuges; ebenso Hans F. Pfenninger, Bedingter Strafvollzug und schweizerische Strafrechtspraxis, SJZ 45/1949, $177 \mathrm{ff}$., 178 f.; wohl auch Rottenberg (Fn. 64), 5 ff.

106 Pieth (Fn. 103), 432, der allerdings anmerkt, dass diese Praxis aufgrund seiner Tarifunsicherheit nicht in ein reines Schuldkonzept passt; a. M. Fellmann (Fn. 50), 92.

107 Vgl. Botschaft, BBl 1999 II 1979 (Fn. 9), 2054.

${ }^{108}$ Statt vieler Roxin (Fn. 66), § 3 N 54.

$109 \mathrm{Vgl}$. Untermassverbot bei BSK StGB-Heer (Fn. 12), Art. 56 N 37; vgl. ferner BGE 107 IV 20 E. 5.c.

110 Schönke/Schröder-Stree/Kinzig (Fn. 79), §56 N 4; Lüthy (Fn. 19), 16.

111 Vgl. BGE 137 IV 72 E. 2.4. 
49 Vor diesem Hintergrund scheint es grundsätzlich konsequent, dass die Dauer der Probezeit die individuell verwirklichte Schuld nicht widerspiegeln muss. ${ }^{12}$ Die Probezeit soll dabei umso länger ausfallen, je grösser die Gefahr der Rückfälligkeit ist. ${ }^{113} \quad$ Nichtsdestotrotz bleibt die bedingte Strafe eine Strafe.

\section{Grenzen des Eingriffs}

5o Die wohl h. L. geht davon aus, dass eine bedingte Strafe verbunden mit Weisungen nicht stärker in die Rechte des Verurteilten eingreifen darf, als es die entsprechende unbedingte Strafe tun würde. ${ }^{114}$ Pieth formuliert dies treffend, jedoch auf den bedingten Strafvollzug allgemein bezogen: «[...] auch 'Gerechtigkeit' und 'nützliche Strafe' im Sinne der Klassiker setzen strenge Schuldadäquanz voraus.»115 Das Bundesgericht scheint

$112 \overline{\text { BSK StGB-Schneider/Garré (Fn. 12), Art. } 44 \mathrm{~N} 4}$; vgl. auch Paul Logoz, Commentaire du Code Pénal Suisse, Partie générale (Art. 1 à 110), Neuchâtel 1939, 184; a. M. Thormann/von Overbeck (Fn. 13), Art. 41 N 12; ein solches System («Schuldmodell») lediglich beschreibend Pieth (Fn. 103), 425.

113 BSK StGB-Schneider/Garré (Fn. 12), Art. 44 N 4.

114 Stratenwerth (Fn. 25, AT II), § 5 N 73; BSK StGB-Schneider/Garré (Fn. 12), Art. 44 N 30; Schultz (Fn. 13, AT II), 111 ff.; Zingg (Fn. 88), 33 f.; Fellmann (Fn. 5O), 91 ff.; Lüthy (Fn. 19), 68; a. M. Johann Riedl, Die Auflagen und Weisungen im Rahmen der Strafaussetzung zur Bewährung, Diss. Tübingen 1972, 72; ebenso Ursula Frauenfelder, Die ambulante Behandlung geistig Abnormer und Süchtiger als strafrechtliche Massnahme nach Art. 43 und 44 StGB, Diss. Zürich 1978, 151, jedoch bezogen auf Weisungen bei Strafaufschub zugunsten von ambulanten Massnahmen.

115 Pieth (Fn. 103), 432 f., welcher im darauffolgenden Satz festhält, dass aktuell gerade das Gegenteil der Fall sei; vgl. auch schon Bruns (Fn. 33), 205: "Auch die Spezialprävention muß sich dem Rechtsstaat beugen! Die Resozialisierung des Verbrechers durch kriminalrichterliche Erziehung, also Auflagen, muß mit ähnlichen Sicherheitsgarantien ausgestattet werden wie die Verhängung von Strafen und die Anordnung von Maßregeln.» diesen Grundsatz zumindest implizit (und allenfalls unbewusst) anzuerkennen, wenn es verlangt, dass Weisungen durch die Art und Schwere der begangenen Tat aufgewogen werden. ${ }^{116}$

51 Die von der Lehre formulierte absolute Grenze muss aus zwei Gründen eingehalten werden: Erstens wird der bedingte Strafvollzug damit gerechtfertigt, dass er milder ist als die ansonsten vollzogene Strafe. ${ }^{117}$ Diese Rechtfertigung löst sich auf, wenn die bedingte Strafe mit ihren Begleiterscheinungen die Eingriffsschwere der unbedingten Strafe erreicht.

Zweitens verliesse das Gericht damit den Bereich der schuldangemessenen Sanktion, was nur dann gerechtfertigt wäre, wenn die Allgemeinheit darüber hinaus vor der Gefährlichkeit des Verurteilten geschützt werden müsste. ${ }^{118}$ Eine solche Gefährlichkeit kann nicht vorliegen, hätte ansonsten der bedingte Strafvollzug doch gar nicht gewährt werden dürfen. So wird im Ergebnis auch die Schwere der begangenen Straftat berücksichtigt und zwar als Eingriffsobergrenze.

Es verbleiben damit aber als Grenzen im Einzelfall nur die Prognose beim bedingten Strafvollzug und die unklare Frage, ob die bedingte Strafe - inkl. Probezeit und Weisungen - die Eingriffsschwere der entsprechenden unbedingten Strafe erreicht. Dass eine Bestimmung der Eingriffsschwere schwierig und nie punktgenau möglich ist, befreit aber nicht davon, zumindest diesbezügliche Erwägungen anzustellen. Auch könnte man sich

\footnotetext{
116 BGE 107 IV 88 E. 3.a.

11 So BGE 137 IV 72 E. 2.4; siehe auch Fellmann (Fn. 50), 93.

118 Roxin (Fn. 66), § 3 N 63.
} 
allenfalls an den für Art. $63 b$ Abs. 4 StGB (Anrechnung des mit einer ambulanten Behandlung verbundenen Freiheitsentzugs) entwickelten Ansätzen orientieren. ${ }^{119}$

Eine solche Abwägung findet sich in der bundesgerichtlichen Praxis jedoch nicht. ${ }^{120}$ Das Bundesgericht stellt zwar fest, dass Weisungen von Betroffenen «subjektiv unter Umständen als ein grösseres Übel empfunden werden als eine unbedingte Strafe», relativiert dies aber damit, dass die mit einer Weisung verbundene bedingte Strafe von der Konzeption des Gesetzes her objektiv weniger schwer wiege. ${ }^{121}$ Dass bedingte Strafen verbunden mit Weisungen schwerer wiegen können als ihr unbedingtes Pendant, dürfte mit den am 1. Januar 2018 wieder eingeführten kurzen Freiheitsstrafen ${ }^{122}$ noch wahrscheinlicher werden.

Unbefriedigend ist auch, dass beim Widerruf der bedingten Strafe keinerlei Anrechnung der bereits verstrichenen Probezeit erfolgt (bspw. analog zu Art. $63 \mathrm{~b}$ Abs. 4 StGB), wodurch der Täter u. U. insgesamt härter sanktioniert wird als derjenige, der (bei gleicher Strafhöhe) von Anfang an eine schlechte Prognose und damit «nur» eine unbedingte Strafe erhalten hat. Dies gilt umso mehr, wenn jener Weisungen zu befolgen hatte.

Dass dieser Zweig der Rechtfertigung des Weisungssystems nicht zu Ende gedacht wurde, zeigt sich bei der Betrachtung der

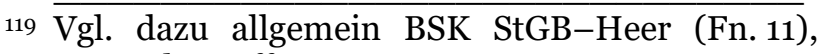
Art. $63 b \mathrm{~N} 6 \mathrm{ff}$.

120 Siehe BGE 94 IV 11 ff.; BGE 130 IV 1, 2 ff.; Urteil des Bundesgerichts 6B_1339/2016 vom 23. März 2017, E. $1 \mathrm{ff}$.

121 BGE 137 IV 72 E. 2.4.

122 AS 20161249. bedingten Geldstrafe. ${ }^{123}$ Die Schwelle der Schwere der hypothetisch vollzogenen Sanktion müsste hier umso schneller erreicht werden, handelt es sich doch «nur» um eine rein finanzielle Einbusse, welche wohl nur in Ausnahmefällen an die Schwere eines Freiheitsentzuges heranreicht. M. E. sollten einzelne Weisungen - wie bspw. eine mehrjährige ambulante Behandlung - bei einer bedingten Geldstrafe grundsätzlich ausgeschlossen sein sollten. ${ }^{124}$

57 Das Institut der Weisung ist im Hinblick auf die Freiheitsstrafe konzeptioniert worden, wurde die Geldstrafe doch erst 2007 eingeführt. Sowohl beim bedingten als auch beim unbedingten Vollzug der Freiheitsstrafe (bedingte Entlassung) kann mit Weisungen auf den Verurteilten eingewirkt werden. Für die Geldstrafe besteht hingegen kein ganzheitliches System. Wer zu einer unbedingten Geldstrafe verurteilt wird, hat mit Bezahlung derselben seine Schuld beglichen. Wer allerdings eine bessere Prognose erhält und zu einer bedingten Geldstrafe verurteilt wird, dem können Weisungen für eine Probezeit von bis zu fünf Jahren auferlegt werden.

123 Diese Problematik wird auch in der Lehre nur stiefmütterlich behandelt, nicht erwähnt bei BSK StGB-Imperatori (Fn. 12), Art. 94; $\mathrm{PK}^{3}-$ Trechsel/Pieth (Fn. 21), Art. 42, 44; PK3Trechsel/Aebersold (Fn. 21), Art. 94; vgl. auch BSK StGB-Schneider/Garré (Fn. 12), Art. 42 $\mathrm{N}$ 84, die nur von Freiheitsstrafe sprechen.

124 Vgl. Günter Stratenwerth, Die Strafen im Bagatellbereich nach künftigem Recht, in: Peter Aebersold/Peter Ablrecht/Felix Bommer/Sabine Gless/Mark Pieth/Martin Schubarth/Kurt Seelmann/Wolfgang Wohlers (Hrsg.), Beiträge zu Grundfragen eines zeitgemässen Strafrechts, Bern 2017, 553, «eine leicht das Absurde streifende Überlegung», zur Kombination von bedingter Geldstrafe und Weisung. 
Dazu kommt, dass sich die Dauer der Probezeit aktuell nicht nach der Strafhöhe bemisst, sondern nach der Persönlichkeit und der Rückfallgefahr des Beschuldigten. ${ }^{125}$ Dies führt nach der gängigen Rechtsprechung im Ergebnis zu der absurden Situation, dass je länger die Probezeit ausfällt, desto schwerer Weisungen in die Rechte des Betroffenen eingreifen dürfen. ${ }^{126}$ Gerade bei Geldstrafen ist bei einer geringen Anzahl Tagessätzen kaum eine Weisung denkbar, welche ausgesprochen auf fünf Jahre nicht der Intensität der unbedingten Geldstrafe gleichkäme. ${ }^{127}$

Nun könnte man einwenden, dass derjenige, welcher die unbedingte Strafe vorgezogen hätte, schlicht die Weisungen missachten und damit auf Vollzug der Strafe drängen könnte und wohl auch wird. ${ }^{128}$ Ein solches «Opting-outSystem» wäre m. E. aber nicht aufrichtig. Auch würde dieses System dem Sinn des bedingten Strafvollzuges widersprechen, der ja den Vollzug gerade vermeiden will. ${ }^{29}$ Ehrlicher wäre es dann, die Weisungen und den bedingten Strafvollzug von vornherein von der Zustimmung des Betroffenen abhängig zu machen. ${ }^{130}$

125 BSK StGB-Schneider/Garré (Fn. 12), Art. 44 N 4.

126 Eine Ausnahme bildet BGE 127 IV 145 E. 2.c.aa, wo festgehalten wurde, dass bei der Festsetzung der Probezeit allfällige Eingriffe durch Weisungen mitberücksichtigt werden müssen. Im Ergebnis wäre es aber sinnvoller, die Weisung nicht für die ganze Probezeit zu erteilen, anstatt die Dauer der Probezeit der Weisung anzupassen.

127 Vgl. Stratenwerth (Fn. 25, AT II), § 5 N 60, «einschneidende Beschränkungen [...], die weitaus schwerer wiegen können als der Vollzug einer Geldstrafe».

128 So schon Lüthy (Fn. 19), 68.

$129 \mathrm{Vgl}$. Fellmann (Fn. 50), $100 \mathrm{f}$.

130 Siehe für ärztliche und psychologische Betreuung hinten V.1.d)

\section{Abgrenzung Massnahme - Weisung}

6o Die unklare und offene gesetzliche Regelung der Weisung erschwert in der Praxis die Abgrenzung des Anwendungsbereichs gegenüber Massnahmen.

\section{Ambulante Massnahme vs. Weisung «Betreuung»}

61 Die grösste Unklarheit scheint im Verhältnis der ambulanten Massnahme nach Art. 63 StGB zu Weisungen bezüglich ärztlicher und psychologischer Betreuung nach Art. 94 StGB zu liegen.131 So kam während der Beratungen der Expertenkommission die Frage auf, inwiefern sich eine ambulante Therapie in Freiheit (als Massnahme) von einer Weisung bezüglich therapeutischer Behandlung bei bedingtem Strafvollzug unterscheide. Eine klare Antwort wurde darauf nicht gefunden. ${ }^{132}$

62 Als Unterschiede der beiden Institute wird oft genannt, dass die Weisung auf die Probezeit beschränkt sei und bei der ambulanten Massnahme eine Umwandlungsmöglichkeit in eine stationäre Form bestehe. Ausserdem müsse für die Anordnung einer Weisung kein besonderer Zustand i. S. der Art. 59 ff. StGB vorliegen.133 Diese Unterschiede ergeben sich allerdings bereits aus dem Gesetz und sagen wenig über das Verhältnis von Weisungen und Massnahmen aus.

63 Für den Verurteilten gibt es zwischen beiden Varianten zunächst einmal keinen

\footnotetext{
131 Vgl. Stratenwerth (Fn. 25, AT II), § 5 N 79.

132 Siehe zum Ganzen Protokolle, Expertenkommission (Fn. 6), $221 \mathrm{ff}$.

133 Zum Ganzen Urteil des Bundesgerichts 6B_1339/2016 vom 23. März 2017, E. 1.1.2; so auch schon Urteil des Bundesgerichts 6S.244/1990 vom 1. August 1990, E. 1.b.
} 
direkt spürbaren Unterschied, solange die ambulante Massnahme mit einem Strafaufschub verbunden wird. ${ }^{134}$ Massnahme und Weisung stünden dabei nicht notwendigerweise in einem Konkurrenzverhältnis, da die Weisung bspw. auch erteilt werden könne, wenn die Voraussetzungen für eine ambulante Massnahme nicht erfüllt seien. ${ }^{135}$

Spricht das Gericht eine Geldstrafe oder eine Freiheitsstrafe von maximal zwei Jahren aus und möchte es, dass der Verurteilte erstens in Freiheit lebt und sich zweitens einer Therapie unterzieht, dann hat es heute grundsätzlich zwei Möglichkeiten: Es kann eine ambulante Massnahme anordnen und den Vollzug der Freiheitsstrafe aufschieben (Art. 63 Abs. 2 StGB) oder eine bedingte Strafe aussprechen und Weisungen erteilen (Art. 44 Abs. 2 i. V. m Art. 94 StGB).

65 Fraglich ist, ob sich die Anwendungsbereiche überhaupt überschneiden und das Gericht nach Ermessen zwischen beiden Optionen wählen kann. Zunächst wird die Situation der Anordnung einer ambulanten Massnahme unter Strafaufschub betrachtet.

\section{a) Ambulante Massnahme unter Strafaufschub}

66 Für therapeutische Massnahmen besteht eine Fülle von strengen allgemeinen (Art. 56 ff. StGB) und speziellen Voraus-

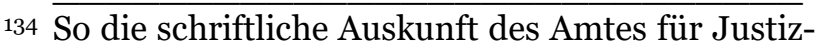
vollzug des Kantons Zürich vom 31. Januar 2017; in diesem Sinne auch BSK StGB-Heer (Fn. 12), Art. 63 N 2, «[...] das Szenario der bedingten Freiheitsstrafe (ev. mit Weisung), das sich von einer ambulanten Massnahme in Freiheit von der Begründung und vom Ablauf her nicht wesentlich unterscheidet $[\ldots] »$.

135 So BSK StGB-Imperatori (Fn. 12), Art. 94 N 17. setzungen (Art. 59, 60 und 61 StGB), welche i. d. R. einen erheblichen Begründungsaufwand mit sich bringen. Verlangt wird dabei u. a. ein besonderer $\mathrm{Zu}$ stand $^{136}$ des Täters. Aus diesem Zustand muss sich eine Sozialgefährlichkeit ergeben, welche sich in der Anlasstat niedergeschlagen hat und welche die Gefahr weiterer Straftaten birgt. ${ }^{137}$ Die jeweilige Gefährlichkeit bemisst sich nach der Schwere der möglichen Delikte und ihrer Eintretenswahrscheinlichkeit (Art. 56 Abs. 2 StGB). ${ }^{138}$ Diese Voraussetzungen gelten grundsätzlich ebenfalls für die ambulante Behandlung. 139

${ }^{6}$ Erst wenn diese Voraussetzungen erfüllt sind, kann über den Aufschub der Freiheitsstrafe entschieden werden (Art. 63 Abs. 2 StGB). ${ }^{140}$ Ein Aufschub einer Geldstrafe wäre aufgrund der klaren gesetzlichen Beschränkung auf Freiheitsstrafen unzulässig. Heer schätzt, dass sich Gerichte immerhin in etwa drei Viertel der Fälle für einen Strafaufschub entscheiden. ${ }^{141}$ Trotzdem komme dem Strafaufschub Ausnahmecharakter zu, ${ }^{142}$ was der bundesgerichtlichen Praxis entspricht. ${ }^{143}$

136 BSK StGB-Heer (Fn. 12), Art. 56 N 17.

137 BSK StGB-Heer, (Fn. 12), Art. $56 \mathrm{~N} 18$.

${ }_{138} \mathrm{Vgl}$. auch BSK StGB-Heer (Fn. 12), Art. 56 $\mathrm{N}$ 37a f.

139 BSK StGB-Heer (Fn. 12), Art. 63 N 24 ff.

140 Ein Aufschub einer Geldstrafe wäre aufgrund der klaren gesetzlichen Beschränkung auf Freiheitsstrafen unzulässig, so BSK StGB-Heer (Fn. 12), Art. $63 \mathrm{~N} 2$ und 34; anders jedoch Urteil des Obergerichts Zürich SB140422 vom 4. Mai 2016, Dispositivziff. 2.b, welches einen Aufschub der gemeinnützigen Arbeit über Art. 63 StGB anordnete.

${ }_{141}$ BSK StGB-Heer (Fn. 12), Art. 63 N 3.

142 BSK StGB-Heer (Fn. 12), Art. 63 N 39; Stratenwerth (Fn. 25, AT II), § 9 N 61.

143 Urteil des Bundesgerichts 6B_107/2011 vom 23. Mai 2011, E. 5.2: «Ein Aufschub rechtfertigt sich aber nur, wenn die ambulante Therapie (ausserhalb des Strafvollzugs) im konkreten Einzelfall 
Täter als ungefährlich eingestuft wird, was i. d. R. ein Gutachter beurteilt. ${ }^{144}$ Ungefährlich bedeutet in diesem $\mathrm{Zu}-$ sammenhang, dass keine hinreichende Wahrscheinlichkeit von Gewaltverbrechen ersichtlich ist. ${ }^{145}$

69 Weiter ist erforderlich, dass die Massnahme vordringlich ist und ihr Erfolg durch einen Freiheitsentzug ernstlich gefährdet würde. ${ }^{146}$ Diese Voraussetzungen unterscheiden sich somit deutlich von denjenigen des bedingten Strafvollzuges. Auch die Legitimationsgrundlage des Eingriffs ist eine andere, nämlich die Sozialgefährlichkeit des Täters. ${ }^{147}$

In Anbetracht der Tatsache, dass dem Strafaufschub zahlenmässig jedoch kaum mehr ein Ausnahmecharakter zugesprochen werden kann, muss wohl unterstellt werden, dass das Institut, wenn die Voraussetzungen für eine Massnahme einmal erfüllt sind, ebenfalls zur Vermeidung schädlicher Freiheitsstrafen genutzt wird, unabhängig von der medizinischen Indikation. ${ }^{148}$ Damit unterscheiden sich die tatsächlichen Voraussetzungen des

aktuelle und günstige Bewährungsaussichten eröffnet, die durch den Strafvollzug zunichtegemacht oder erheblich vermindert würden »; siehe auch BGE 129 IV 161 E. 4.1.

144 BSK StGB-Heer (Fn. 12), Art. 63 N 41 ff.

145 Vgl. BSK StGB-Heer (Fn. 12), Art. 63 N 43; auch Jörg Rehberg, Fragen bei der Anordnung und Aufhebung sichernder Massnahmen nach StrGB Art. 42-44, ZStrR 93/1977, 164 ff., 167 ff., 176.

146 BGE 101 IV 270 E. 1; BSK StGB-Heer (Fn. 12), Art. $63 \mathrm{~N} 47 \mathrm{f}$.

147 BSK StGB-Heer (Fn. 12), Art. 56 N 18.

148 Das Bundesgericht bedient sich hierzu eines Kunstgriffs, indem es die Resozialisierungschancen bei einem Strafaufschub als Teil der Therapie betrachtet. So geht bei guter Prognose die Therapie dem Strafvollzug vor. Siehe dazu BGE 120 IV 1 E. 2.b; siehe auch BSK StGB-Heer (Fn. 12), Art. 63 N 48 .
Strafaufschubs im Ergebnis kaum von denjenigen der bedingten Strafe.

\section{b) Anwendbarkeit bei bedingtem Strafvollzug}

${ }^{71}$ Auch wenn die Argumentation beim Strafaufschub zugunsten einer Massnahme derjenigen des bedingten Strafvollzuges nahekommt, hat der Gesetzgeber die Hürde für Massnahmen, auch wenn diese ambulant vollzogen werden sollen, absichtlich hoch angesetzt. Steht bei einer beschuldigten Person der bedingte Strafvollzug im Raum, dann können die Voraussetzungen für eine therapeutische Massnahme nicht erfüllt sein. Das Bundesgericht hat dies zunächst nur für die stationären Massnahmen festgestellt, 149 in späteren Entscheiden jedoch auch ausdrücklich für ambulante Massnahmen bestätigt. ${ }^{150}$

72 Dies erscheint insofern konsequent, als dass sich die Prognosen bei stationären und ambulanten Massnahmen nicht unterscheiden, sondern vielmehr die medizinische und therapeutische Indikation über die Vollzugsform entscheidet. Die Anordnung einer therapeutischen Massnahme schliesst den bedingten Strafvollzug aus, weil sie eine schlechte Prognose voraussetzt, was in unüberwindbarem Widerspruch zur Prognose beim bedingten Strafvollzug steht. ${ }^{151}$ Folglich ist eine

$149 \overline{\text { BGE } 135 \text { IV } 180 \text { E. 2.3; vgl. PK3-Trechsel/Pieth }}$ (Fn. 21), Art. 42 N 5; auch Schultz (Fn. 13, AT II), 106.

150 Zuletzt Urteil des Bundesgerichts 6B_652/2016 vom 28. März 2017, E. 3.3.1; Urteil des Bundesgerichts 6B_1227/2015 vom 29. Juli 2016, E. 1.2.4; ferner BGE 135 IV 180 E. 2.3; vgl. auch BSK StGB-Heer (Fn. 12), Art. 59 N 118.

${ }_{151}$ Urteil des Bundesgerichts 6B_652/2016 vom 28. März 2017, E. 3.3.1; so schon Frauenfelder (Fn. 114), 96 ff.; vgl. auch ZBJV 111 (1975) 87 f.; zu stationären Massnahmen siehe BGE 135 
ambulante Massnahme nur bei unbedingten Strafen möglich. ${ }^{152}$ Ist eine ambulante Massnahme notwendig, dann ist die Strafe immer unbedingt auszusprechen. ${ }^{153}$

Damit besteht zumindest auf dem Reissbrett zwischen therapeutischen Massnahmen und Weisungen betreffend ärztliche und psychologische Betreuung kein Konkurrenzverhältnis. Massnahmen sind zwingend anzuordnen, wenn ihre Voraussetzungen erfüllt sind. ${ }^{154}$ Konsequenterweise ist es deshalb bspw. unzulässig, bei Massnahmebedürftigkeit stattdessen eine Weisung zur Alkoholabstinenz zu erteilen. ${ }^{155}$

74 Damit ist dem Gericht in der Praxis aber kaum geholfen, wenn bspw. eine geringe Geldstrafe im Raum steht, aber eindeutig eine Massnahmebedürftigkeit besteht. Es muss sich in diesen Bagatellfällen wohl aus Gründen der Verhältnismässigkeit auch weiterhin für eine bedingte Strafe mit Weisung entscheiden, auch wenn eine schlechte Prognose vorliegt. Eine bessere Lösung bietet das Gesetz aktuell nicht.

IV $180 \quad$ E. 2.3; Schwarzenegger/Hug/Jositsch (Fn. 24), 132; BSK StGB-Heer (Fn. 12), Art. 59 N 118, Art. 63 N 90; vgl. ferner Rehberg (Fn. 145), 176.

152 Vgl. BSK StGB-Heer (Fn. 12), Art. 63 N 2; inwiefern Massnahmen auch bei Geldstrafen gerechtfertigt werden können, kann in dieser Arbeit nicht vertieft werden; anders aber Urteil des Kantonsgerichts Freiburg vom 8. Juni 2016, 5012015 49, Ziff. 5 und 6.

153 Zuletzt Urteil des Bundesgerichts 6B_652/2016 vom 28. März 2017, E. 3.3.1.

154 Statt vieler BSK StGB-Heer (Fn. 12), Art. 56 N 15, 90; Urteil des Bundesgerichts 6B_1227/2015 vom 29. Juli 2016, E. 1.2.4.

155 Urteil des Obergerichts Zürich SB160094 vom 27. Juni 2016, Ziff. 4.

\section{c) Weisungen betreffend ärztliche und psychologische Betreuung}

75 Fraglich ist, ob die Nichtanwendbarkeit von therapeutischen Massnahmen bei bedingtem Strafvollzug bedeutet, dass dieselbe Behandlung über eine Weisung angeordnet werden kann. Bei Unterscheidung von Massnahme und Weisung wird häufig ins Feld geführt, dass Weisungen insbesondere dann erteilt werden können, wenn die Voraussetzungen von Massnahmen nicht vorliegen. ${ }^{156}$ Diese Feststellung ist nach aktueller Rechtslage grundsätzlich richtig, jedoch besteht zwischen dem Fehlen von Massnahmenvoraussetzungen und der Zulässigkeit von Weisungen keine direkte Verbindung. ${ }^{157}$ Fehlt eine Voraussetzung der Massnahme, wird dadurch die Legalprognose nicht direkt besser und der bedingte Strafvollzug nicht per se anwendbar. Entscheidend für eine Weisung kann also nicht sein, ob eine Massnahme an einer Voraussetzung (wie bspw. der schweren psychischen Störung oder auch der Verhältnismässigkeit) scheitert, sondern nur die Legalprognose. Denkbar wäre also, dass die Gefährlichkeit für eine therapeutische Massnahme nicht ausreicht.

Wenig problematisch sind diejenigen Weisungen bezüglich ärztlicher oder psychologischer Betreuung, welche eine deutlich geringere Eingriffsschwere als eine ambulante Behandlung nach Art. 63 StGB aufweisen. Dies betrifft vor allem neue, niederschwellige Formen von The-

$1 5 6 \longdiv { \text { So etwa BSK StGB-Imperatori (Fn. 12), Art. } 9 4 }$ $\mathrm{N} 17$.

157 Anders noch die Rechtsprechung bevor das Bundesgericht den gegenseitig ausschliessenden Charakter von therapeutischen Massnahmen und Weisungen anerkannte, vgl. bspw. Urteil des Bundesgerichts 6S.244/1990 vom 1. August 1990, E. 1.b ff. 
rapien, wie bspw. Lernprogramme für Täter im Kontext von häuslicher Gewalt, die dem Täter ein Alternativverhalten für Konfliktsituation näherbringen sollen.158

77 Doch ist es allgemein zulässig, Weisungen zu erteilen, welche sich inhaltlich mit einer ambulanten Massnahme nach Art. 63 StGB decken?

In der Lehre wird zum Verhältnis von Massnahmen und Strafen zu anderen, ähnlichen Interventionsmöglichkeiten eine allgemeine Diskussion unter dem Titel «Sperrwirkung von Strafen und Massregeln» 159 geführt. Weisungen dürften danach nicht den Inhalt von Strafen und Massnahmen haben, da letztere i. d. R. strengeren Voraussetzungen unterliegen, welche gesondert geregelt sind. Liegen diese Voraussetzungen nicht vor, schirmen sie den Beschuldigten auch negativ ab, das heisst, der als Massnahme vorgesehene Eingriff kann nicht anderweitig angeordnet werden. ${ }^{160}$ Ein Strafrecht, welches den Grundsatz «keine Sanktion ohne Gesetz» ernst nimmt, muss die Sperrwirkung von Strafen und Massnahmen beachten.

Allerdings liegt mit dem bedingten Strafvollzug eine Situation vor, auf welche die Theorie der Sperrwirkung von Strafen und Massregeln nicht gut übertragen

158 Siehe BSK StGB-Imperatori (Fn. 12), Art. 93 $\mathrm{N}$ 28; Bericht des Bundesamtes für Justiz in Erfüllung des Postulats 11.4072 Amherd vom 15. Dezember 2011: Überprüfung des Straf- und Massnahmenvollzuges in der Schweiz, Bern 18. März 2014, 29.

159 Vgl. dazu Fellmann (Fn. 50), 131 f.; Bruns (Fn. 33), 222 f.; ferner Schönke/Schröder-Stree/ Kinzig (Fn. 79), § 56c N 12.

160 Siehe Fellmann (Fn. 50), 131 f.; Bruns (Fn. 33), 222 f.; $\quad$ a. M. Schönke/Schröder-Stree/Kinzig (Fn. 79), § 56c N 12, welche Weisungen sehr weitgehend zulassen wollen. werden kann. Die schuldangemessene (und damit als gerechtfertigt angesehene) ${ }^{161}$ Strafe wird festgesetzt, ihr Vollzug aber aus spezialpräventiven Gründen aufgeschoben. Dass damit die Sanktion faktisch geringer ausfällt, als das Mass der Schuld erlauben würde, gibt straftheoretisch zu keiner Kritik Anlass. ${ }^{162}$ Mit Weisungen kann dieses «Sanktionsdefizit» gewissermassen bis zur schuldadäquaten Sanktion «kompensiert» werden. ${ }^{163}$ Da sich das Gericht mit Weisungen also im schuldadäquaten Sanktionsrahmen zu bewegen hat, spricht grundsätzlich nichts gegen eine Anordnung einer Therapie, welche auch über Art. 63 StGB möglich wäre - solange diese prognoserelevant ist. ${ }^{164}$ Dies bedingt natürlich, dass die Weisung dennoch verhältnismässig ist. Hier liegt der eigentliche Kern des Problems.

so Zunächst muss die Behandlung überhaupt geeignet sein, die Rückfallgefahr zu senken bzw. die Resozialisierung zu fördern. Die Anordnung einer Psychotherapie oder einer medikamentösen Behandlung sollte sich deshalb auf ein Gutachten stützen. ${ }^{165}$ So kann auch vermieden werden, dass einzelne Weisungen «den Eindruck einer Alibi-Übung»166 erwecken. Liegt kein Gutachten vor, muss bezüglich der Indikation der Therapie

161 Vgl. dazu Stratenwerth (Fn. 25, AT II), § 8 N 2.

162 Roxin (Fn. 66), § $3 \mathrm{~N} 54$. Zu beachten ist hier, dass es sich beim bedingten Strafvollzug nicht um eine Unterschuldstrafe im technischen Sinne handelt, höchstens im faktischen.

163 Beachte aber, dass dies aufgrund fehlender Anrechnung nicht für den Widerrufsfall gilt.

164 Vgl. vorne II.1.c) und d).

165 So auch BSK-Schneider/Garré (Fn. 12), Art. 44 $\mathrm{N}$ 44, welche aber etwas «blauäugig» davon auszugehen scheinen, dass die Gerichte sich selbst zur Einholung von Gutachten verpflichten.

166 So BSK StGB-Heer (Fn. 12), Art. 63 N 30, jedoch zur ambulanten Massnahme. 
zumindest einiges an Abklärungs- und Begründungsaufwand betrieben werden. ${ }^{167}$ Auch ist mehr Augenmerk darauf zu richten, ob sich einzelne Therapieformen isoliert überhaupt als Weisung eignen. ${ }^{168}$ Schliesslich ist bei Unklarheit, ob eine Massnahmebedürftigkeit vorliegt, zunächst die Notwendigkeit einer Massnahme zu prüfen, was i.d.R. ohnehin mit Gutachten erfolgt. ${ }^{169}$

Die Weisung muss erforderlich sein und damit auch in zeitlicher Hinsicht auf die notwendige Dauer beschränkt werden, unabhängig davon, wie lange die Probezeit ausfällt. ${ }^{170}$

Zur Zumutbarkeit gilt es den Gedanken der Interessenabwägung wieder aufzunehmen und noch einmal festzuhalten, dass es sich beim Ziel der «Besserung» primär um ein öffentliches Interesse handelt. ${ }^{171}$ Es ist ein "gesellschaftliche[s] Interesse an einer Veränderung der Persönlichkeitsstruktur [...] des Betroffenen». ${ }^{172}$ Dies gilt auch für die medikamentöse Behandlung, denn diese greift ebenfalls in den Kern der Persönlichkeit ein, jedoch hauptsächlich in deren körperlichen Aspekt. Dadurch unterscheidet

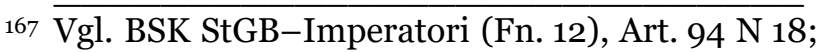
Urteil des Bundesgerichts 6A.36/2003 vom 6. Juni 2003, E. 2.

168 Was insbesondere bei der Antabusbehandlung in Zweifel gezogen wird. Siehe dazu BSK StGBHeer (Fn. 12), Art. 63 N 7.

169 Vgl. dazu Urteil des Bundesgerichts 6B_652/2016 vom 28. März 2017, E. 3.4.1 f.

${ }_{170}$ Vgl. dazu BSK StGB-Schneider/Garré (Fn. 12), Art. $44 \mathrm{~N} 19$.

${ }_{171} \mathrm{Vgl}$. vorne II.2.b).

${ }_{172}$ Stratenwerth (Fn. 25, AT II), § 8 N 5, jedoch auf die Besserung bei Massnahmen bezogen. Der Gedanke lässt sich aber auf die entsprechenden Weisungen übertragen. Vgl. ferner BSK StGB-

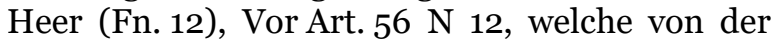
«Erzeugung einer 'rechtskonformen' Persönlichkeit» spricht. sich die Therapie von den restlichen Weisungen. Es handelt sich dabei um einen Eingriff in den Kern der Persönlichkeit, dem sich eine Person nur unter Einhaltung höchster Anforderungen sollte unterziehen müssen, weshalb der Gesetzgeber die allgemeinen Massnahmenvoraussetzungen (Art. $56 \mathrm{ff}$. StGB) geschaffen hat. ${ }^{173}$ Bei der Erteilung von Weisungen müssen aber kaum «höchste Anforderungen» erfüllt werden, weshalb man die Ansicht vertreten könnte, dass die Anordnung solcher Therapien über Weisungen generell unverhältnismässig ist.

83 Man muss indes nicht so weit gehen, diese zu verbieten, zumal es einen sinnvollen und zulässigen Anwendungsbereich geben kann. An dieser Stelle kommt das Interesse der beschuldigten Person ins Spiel.

\section{d) Erforderlichkeit des Einverständnisses?}

84 Nach den Grundgedanken und dem Gesetzeswortlaut der ambulanten Massnahme und der Weisung ist das Einverständnis des Verurteilten zu einer Therapie nicht erforderlich. Dieses spielt aber eine erhebliche Rolle, wenn Eingriffe in die Persönlichkeit einer Person zur Diskussion stehen. Einerseits ist eine Behandlung ohne Kooperation des Betroffenen kaum je erfolgversprechend. ${ }^{174}$ Andererseits basieren therapeutische Massnahmen auf der (Rückfall-) Gefährlichkeit von Personen und können deshalb sinnvollerweise nicht von deren Zustimmung abhängig gemacht werden.

\footnotetext{
173 Siehe Botschaft, BBl 1999 II 1979 (Fn. 9), 2069.

174 BSK StGB-Heer (Fn. 12), Art. 63 N 29; Stratenwerth (Fn. 25, AT II), § 5 N 79, § 9 N 65; Frauenfelder (Fn. 114), $246 \mathrm{f}$.
} 
Namentlich muss eine Therapiemotivation oft erst geschaffen werden. ${ }^{175}$

Bei einer Weisung stellt sich aufgrund der zwingend fehlenden Gefährlichkeit (bei zusätzlich höchstens moderater Rückfallgefahr) in diesem Sinne eine andere Ausgangslage: Der Eingriff bedarf einer anderen Rechtfertigung. Das öffentliche Interesse an einer wünschenswerten «Besserung» ohne wirkliche Not im Sinne der Gefährlichkeit kann nicht ausreichen, um einen Eingriff in den Kern der Persönlichkeit zu rechtfertigen. ${ }^{176}$ Anders liegt die Situation, wenn der Eingriff zugleich im Interesse der betroffenen Person liegt, wobei i. d. R. auf das wohlverstandene Interesse abgestellt wird. 177 In einer Gesellschaft, in der das Individuum ernst genommen wird, muss jedoch - wenn immer möglich - auf das faktische Interesse der beschuldigten Person abgestellt werden. ${ }^{178}$

Als Konsequenz sind diese Weisungen (ausnahmsweise) ${ }^{179}$ vom Einverständnis der beschuldigten Person abhängig zu machen. Damit hat der Betroffene die Wahl zwischen bedingtem und unbedingtem Vollzug. Bei einer Geldstrafe wird eine solche Abwägung wohl noch zu be-

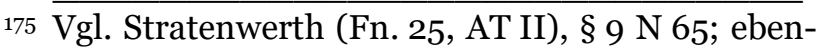
so bezüglich der Lernprogramme, Mösch Payot (Fn. 72), Rz. 25.

176 Vgl. Stratenwerth (Fn. 25, AT II), § 8 N 5, der darin ein unzulässiges Verfügen über den Betroffenen in instrumenteller Weise sieht und dies sogar für stationäre Massnahmen ablehnt; kritisch auch BSK StGB-Imperatori (Fn. 12), Art. 94 N 18; Gisel-Bugnion (Fn. 49), 67 ff.

177 Vgl. statt vieler Stratenwerth (Fn. 25, AT II), § 8 $\mathrm{N}$ 27; sowie vorne II.2.b) und c).

${ }^{178}$ So auch Stratenwerth (Fn. 25, AT II), § 8 N 28, welcher einen Rückgriff auf das wohlverstandene Interesse dann befürwortet, wenn das faktische Interesse gerade durch den zu behandelnden $\mathrm{Zu}$ stand beeinträchtigt wird.

179 Siehe vorne Rz. 31 f. werkstelligen sein, da man den genauen «Preis» der unbedingten Strafe kennt. Steht jedoch eine Freiheitsstrafe im Raum, ist diese Abwägung für den Betroffenen wohl ebenso schwer vorzunehmen wie für das Gericht. Hinzu kommt, dass der Betroffene aus dem Wortlaut der Weisung die Intensität des Eingriffs kaum abschätzen kann. Das Gericht beschränkt sich häufig auf die Formulierung «...hat sich einer psychologischen Behandlung zu unterziehen ${ }^{180}$. Nichtsdestotrotz mag es Personen geben, die eine unbedingte Freiheitsstrafe einer langjährigen Behandlung vorziehen. Diese Entscheidungsfreiheit sollte man ihnen nicht absprechen, da sie diese ohnehin im Sinne des Opting-out ${ }^{181}$ haben. In diese Richtung liefen auch Teile der Diskussion der Expertenkommission, welche die Revision des Allgemeinen Teils von 2007 vorberiet. ${ }^{182}$ Wie Art. 44 und 94 StGB zeigen, ist dieser Ansatz aber nicht Gesetz geworden.

\section{Tätigkeitsverbot vs. Weisung «Berufsausübung»}

87 Das Tätigkeitsverbot ${ }^{183}$ (Art. 67 f. StGB) ist in seiner heutigen Fassung seit dem 1. Januar 2015 in Kraft. Allerdings entsprechen Art. 67 und $67 a$ StGB nur einem vorauseilenden indirekten Gegenvorschlag zur Volksinitiative «Pädophile sollen nicht mehr mit Kindern arbeiten dürfen». Die definitive Umsetzung von Art. 123c BV, wird aktuell im Parlament beraten. ${ }^{184}$

180 Siehe statt vieler Urteil des Bundesgerichts 6B_166/2016 vom 7. Juli 2016, E. 4.1.

${ }^{181}$ Siehe vorne, III.3.

182 Protokolle, Expertenkommission (Fn. 6), 617 ff., 1069 ff.; vgl. auch Gisel-Bugnion (Fn. 49), 67;

${ }_{183}$ Früher als Nebenstrafe des Berufsverbots, siehe BSK StGB-Niggli/Maeder (Fn. 12), Art. 67 N 1.

184 Botschaft vom 3. Juni 2016 zur Änderung des Strafgesetzbuchs und des Militärstrafgesetzes 
Das Gericht kann ein Tätigkeitsverbot nach Art. 67 Abs. 1 StGB aussprechen, wenn jemand in Ausübung einer beruflichen (oder einer organisierten ausserberuflichen) Tätigkeit ein Verbrechen oder Vergehen begangen hat und dafür zu einer Freiheitsstrafe von mindestens sechs Monaten verurteilt wurde. ${ }^{185}$ Somit wird - wie bei den therapeutischen Massnahmen - eine (hier zur Tätigkeit) konnexe Anlasstat verlangt. Weiter wird vorausgesetzt, dass die Gefahr besteht, der Täter werde seine Tätigkeit zur Begehung weiterer Verbrechen oder Vergehen missbrauchen. Eine Tätigkeit kann nach Abs. 1 ganz oder teilweise für sechs Monate bis $\mathrm{zu}$ fünf Jahren verboten werden. ${ }^{186}$ Wer die verbotene Tätigkeit dennoch ausübt, kann nach Art. 294 StGB mit Freiheitsstrafe bis zu einem Jahr oder Geldstrafe bestraft werden.

Das Tätigkeitsverbot weist eindeutig einen spezialpräventiven Charakter auf. ${ }^{187}$ Als spezialpräventive Massnahme muss sich der Eingriff somit an der vom Täter ausgehenden Gefahr messen und nicht an der Schwere der verwirklichten Tat. ${ }^{888}$ Ziel ist es, eine Wiederholung bestimmter mit der Ausübung einer Tätigkeit zusammenhängender Straftaten zu verhindern. ${ }^{189}$

(Umsetzung von Art. 123c BV) (BBl 2016 6115); AB 2017 S 629 ff.;

185 Da die Geldstrafe neu auf max. 180 Tagessätze beschränkt ist (Art. 34 Abs. 1 StGB), ist Art. 67 StGB nur noch auf Freiheisstrafen anwendbar.

186 Die Abs. 2-6 von Art. 67 StGB zielen auf eine Verschärfung der Massnahme zum Schutze von Minderjährigen und anderen besonders schutzbedürftigen Personen $\mathrm{ab}$ und werden an dieser Stelle nicht weiter vertieft.

187 Siehe dazu Botschaft, BBl 1999 II 1979 (Fn. 9), 2104; BSK StGB-Niggli/Maeder (Fn. 12), Art. 67 N 27, «[es geht] ausschliesslich um Gefahrenabwehr (Prävention)».

188 BSK StGB-Niggli/Maeder (Fn. 12), Art. 67 N 27.

189 BSK StGB-Niggli/Maeder (Fn. 12), Art. 67 N 23.

\section{a) Anwendbarkeit bei bedingtem Strafvollzug}

9o Grundsätzlich setzt Art. 67 StGB eine Wiederholungsgefahr voraus («besteht die Gefahr, dass er seine Tätigkeit zur Begehung weiterer Verbrechen oder Vergehen missbraucht»), ${ }^{190}$ was zumindest in einem Spannungsverhältnis zum bedingten Strafvollzug steht. ${ }^{191}$ Ein Tätigkeitsverbot ist andererseits nur sinnvoll, wenn sich der Adressat in Freiheit befindet, er andernfalls gar keine Gelegenheit hat, wieder berufsbedingt zu delinquieren. Auch soll die Möglichkeit des Tätigkeitsverbots gerade $\mathrm{zu}$ einer günstigen Prognose führen können. ${ }^{192}$ Art. $67 \mathrm{c}$ Abs. 3 StGB lässt dann auch kaum einen anderen Schluss zu, als dass das Tätigkeitsverbot auch bei bedingten Strafen zur Anwendung kommt, ${ }^{193}$ hat doch der Gesetzgeber für den Fall des Strafwiderrufs festgelegt, dass das Verbot erst mit der Entlassung zu laufen beginnt.

91 Dies lässt sich jedoch nur schwer mit dem bedingten Strafvollzug vereinbaren. Gerade die Vergehensstrafe (Art. 294 StGB) bei Missachtung des Tätigkeitsverbots scheint dem Grundgedanken des bedingten Vollzuges zu widersprechen.

190 Vgl. auch BSK StGB-Niggli/Maeder (Fn. 12), Art. $67 \mathrm{~N} \mathrm{25}$, «schlechte Legalprognose».

$191 \mathrm{Vgl}$. auch Dupuis et al. (Fn. 51), Art. 67 N 1, «contradiction».

192 Botschaft, BBl 1999 II 1979 (Fn. 9), 2106; BSK StGB-Niggli/Maeder (Fn. 12), Art. 67 N 30; Dupuis et al. (Fn. 51), Art. 67 N 1; Lehner (Fn. 41), 12; PK3-Bertossa (Fn. 21), Vor Art. $67 \mathrm{~N}_{4}$, Art. $67 \mathrm{~N} 5$.

193 So auch die h. L.: Dupuis et al. (Fn. 51), Art. 67 N 1; BSK StGB-Niggli/Maeder (Fn. 12), Art. 67 $\mathrm{N}$ 30; unklar PK3-Bertossa (Fn. 21), Art. 67 N 5; klar bejahend noch die Vorauflage, Stefan Trechsel/Carlo Bertossa, Art. 67, in: Stefan Trechsel/Mark Pieth (Hrsg.), Schweizerisches Strafgesetzbuch, Praxiskommentar, 2. Auflage, Zürich/St. Gallen 2013 (fortan $\mathrm{PK}^{2}$-BearbeiterIn), $\mathrm{N} 3$ 
Bei einem Verstoss gegen eine Weisung stehen dem Gericht bereits verschiedene Optionen nach Art. 95 StGB zur Verfügung. Bei Verstoss gegen ein Tätigkeitsverbot nach Art. 67 StGB erfolgt jedoch zwingend eine Bestrafung nach Art. 294 StGB. Um diese Strafdrohung zu rechtfertigen, muss m. E. eine gegenüber den Voraussetzungen des bedingten Strafvollzugs erhöhte Rückfallgefahr vorliegen. Dieser Prognosewiderspruch lässt sich kaum sinnvoll auflösen.

Ist ein Beschuldigter derart rückfallgefährdet, dass ein Berufsverbot in Erwägung gezogen wird, sollte grundsätzlich kein bedingter Strafvollzug gewährt werden können. Es wäre jedoch absurd, das Gericht zur Verhängung einer unbedingten Strafe zu zwingen, wenn jemand «nur» so spezifisch rückfallgefährdet ist, dass ein Tätigkeitsverbot unabdingbar ist. Dabei hängt die Beurteilung auch davon $\mathrm{ab}$, ob der Beschuldigte für die körperliche, psychische oder vor allem sexuelle Integrität Dritter gefährlich ist. In solchen Fällen kann der bedingte Strafvollzug wohl nur in Ausnahmefällen gewährt werden. Ist in anderen Fällen aus spezialpräventiver Sicht der bedingte Strafvollzug gleich gut oder besser geeignet als der unbedingte Vollzug, dann soll dieser verhängt werden dürfen, selbst wenn eine Massnahme nach Art. $67 \mathrm{ff}$. StGB notwendig ist.

Die Anwendung des Tätigkeitsverbots als Massnahme bei bedingtem Strafvollzug sollte aber nur im Grenzbereich zwischen bedingter und unbedingter Strafe zulässig sein. In diesem Bereich besteht dann jedoch das generelle Problem, dass Strafen zugunsten von Massnahmen aufgeschoben werden können, für welche der
Aufschub gesetzlich gar nicht vorgesehen ist.

94 Fraglich ist hier auch, ob sich die Massnahme ebenfalls an der Eingriffsobergrenze der unbedingten Strafe zu orientieren hat, sobald diese zu einer bedingten Strafe hinzutritt. Dies muss verneint werden, da die Massnahme auch in Verbindung mit einer unbedingten Strafe verhängt werden könnte und dort ohnehin nicht schuldadäquat sein müsste. ${ }^{194}$

\section{b) Verhältnis zur Weisung bezüglich Berufsausübung}

95 In der Lehre werden Massnahmen von ihren Weisungspendants primär anhand des verfolgten Zwecks unterschieden. Hat die Einschränkung der Berufsausübung primär den Schutz Dritter als Ziel, so könne nur eine Massnahme nach Art. 67 StGB in Betracht kommen - so die wohl h. L. ${ }^{195}$ Im Gegenzug wird schlicht behauptet, dass die Weisung bezüglich Berufsausübung (mit identischem Inhalt!) im Interesse des Betroffenen selbst angeordnet wird. ${ }^{196}$ Es ist nicht ersichtlich, inwiefern ein Berufsverbot im einen Fall der Sicherheit Dritter und in einem anderen Fall der Resozialisierung dienen soll. ${ }^{197}$

\footnotetext{
194 Vgl. BSK StGB-Heer (Fn. 12), Vor Art. 56 N 1.

195 Vgl. Stratenwerth (Fn. 25, AT II), \& 5 N 75; Stratenwerth/Wohlers (Fn. 69), Art. $94 \mathrm{~N}$ 3; so wohl auch PK3-Trechsel/Aebersold (Fn. 21), Art. 94 $\mathrm{N} 4$.

196 BSK StGB-Schneider/Garré (Fn. 12), Art. 44 N 36; Mühlemann (Fn. 69), 179 f.; a. M. noch $\mathrm{PK}^{2}$-Trechsel/Bertossa (Fn. 193), Art. 67 N 2; nicht problematisiert in $\mathrm{PK}_{3}-$ Bertrossa, Art. 67; vgl. auch Lehner (Fn. 41), 20; zur Problematik des «wohlverstandenen Interesses» siehe vorne Kap. II.2.b) und IV.1.d).

197 So schon Bruns (Fn. 33), 226: «Wer könnte auch genau abgrenzen, inwieweit ein Berufsverbot der Sicherung oder (und) der Besserung dient und
} 

bedingte Geldstrafe von 3 bis 180 Tagessätzen (Art. 34 Abs. 1 StGB) oder eine Freiheitsstrafe von 3 bis 180 Tagen (Art. 40 Abs. 1 StGB) handeln. Es fällt deshalb schwer, Argumente für die $\mathrm{Zu}-$ lässigkeit eines Tätigkeitsverbots über eine Weisung zu finden. Der Gesetzgeber hat die Schwelle für Art. 67 StGB für nicht zufällig bei einer Freiheitsstrafe von sechs Monaten gesetzt. Mit Beschränkung der Geldstrafen auf maximal 180 Tagessätze hätte er Art. 67 Abs. 1 StGB nach unten öffnen können, um weiterhin eine Kombination mit Geldstrafen zu erlauben. Dies wurde aber nicht gemacht. Damit sollten beschuldigte Personen gegen inhaltlich identische Weisungen negativ abgeschirmt sein und es wäre stossend, wenn die Massnahmenvoraussetzungen im Falle einer bedingten Strafe durch die Anordnung von Weisungen umgangen werden könnten. ${ }^{199}$ Das Verbot einer Tätigkeit ist als schwerer Eingriff in die Wirtschaftsfreiheit und als po-

demgemäß die entsprechenden Auflagen unterschiedlich beurteilen!»

198 Ein anderer Grund für die fehlende Verhältnismässigkeit ist m. E. nicht denkbar, da bspw. die Minimaldauer mit sechs Monaten sogar kürzer bemessen ist als diejenige für die Weisung. Dem Autor ist keine Weisung bekannt, welche die Mindestdauer der Probezeit von zwei Jahren unterschritten hätte.

199 Vgl. allgemein Bruns (Fn. 33), 222 f., für ihn wird damit ein «unerträglicher Etikettenschwindel» betrieben, es handle sich um eine «Gesetzesumgehung», vgl. auch 226; vgl. ferner Fellmann (Fn. 50), 61; a. M. BSK StGB-Schneider/Garré (Fn. 12), Art. $44 \mathrm{~N} 36$. tentiell resozialisierungsgefährdende Intervention auf die gravierendsten Fälle zu beschränken. ${ }^{200}$

98 Weiter ist nicht ersichtlich, inwiefern ein Verbot eines Berufs die Resozialisierung fördern und im Interesse des Betroffenen liegen sollte. ${ }^{201}$ Zur Rechtfertigung einer solchen Weisung müsste man das Erfordernis der resozialisierenden Wirkung wohl endgültig zugunsten der Spezialprävention aufgeben. Die Ansicht, ein umfassendes Verbot von (ausserberuflichen) Tätigkeiten sei auch über Weisungen möglich,202 ist deshalb abzulehnen. ${ }^{203}$

99 Das Tätigkeitsverbot nach Art. 67 StGB regelt die Möglichkeit, eine Tätigkeit als solche $\mathrm{zu}$ verbieten abschliessend. ${ }^{204}$ Dies scheint auch die klarste Lösung zu sein. Allerdings ist eine zwingende Strafandrohung nach Art. 294 Abs. 1 StGB bei bedingtem Strafvollzug nicht sinnvoll. Hier drängt sich eine Anpassung auf.

100 Es verbleibt dort ein Restanwendungsbereich für eine Weisung betreffend Berufsausübung, wo nicht ein Verbot einer Tätigkeit, sondern allenfalls die Art und Weise der Berufsausübung zur Debatte steht, wie bspw. das Verbot des Handels mit Sexartikeln. ${ }^{205}$

200 So Botschaft, BBl 1999 II 1979 (Fn. 9), 2103 f.; im Ergebnis ebenso Mühlemann (Fn. 69), 150 ff.

${ }^{201}$ So noch PK ${ }^{2}-$ Trechsel/Bertossa (Fn. 193), Art. 67 $\mathrm{N}_{1}$; a. M. Lehner (Fn. 41), 86 ff. mit weiteren Hinweisen.

${ }^{202}$ So Botschaft, BBl 20128819 (Fn. 22), 8848; siehe auch Schultz (Fn. 13, ZStrR), 60 f.

203 Gl. M. Fellmann (Fn. 50), 119 f.; Mühlemann (Fn. 69), 178, bezüglich des Verbots einer bestimmten Art von Erwerbstätigkeit; kritisch auch Stratenwerth (Fn. 25, AT II), § 5 N 75.

204 Wohl zustimmend PK3-Trechsel/Aebersold (Fn. 21), Art. $94 \mathrm{~N} \mathrm{4}$, jedenfalls für den Anwendungsbereich von Art. 67 StGB.

205 BGE 105 IV $289 \mathrm{f}$. 


\section{Kontakt- und Rayonverbot vs. Weisung «Aufenthalt»}

101 Das Kontakt- und Rayonverbot (Art. $67 b \mathrm{ff}$. StGB) ist zusammen mit dem Tätigkeitsverbot nach Art. 67 StGB eingefügt worden. ${ }^{206}$ Es trat am 1. Januar 2015 in Kraft.207 Der Fokus lag aber nicht nur auf Sexualstraftätern, sondern vor allem auf Konstellationen von Stalking und häuslicher Gewalt. ${ }^{208}$ Der Wortlaut von Art. $67 \mathrm{~b}$ Abs. 1 StGB ist entsprechend offen: «Hat jemand ein Verbrechen oder Vergehen gegen eine oder mehrere bestimmte Personen [...] begangen und besteht die Gefahr, dass er bei einem Kontakt zu diesen Personen weitere Verbrechen oder Vergehen begehen wird, so kann das Gericht für eine Dauer bis zu fünf Jahren ein Kontakt- und Rayonverbot verhängen». Art. $67 b$ StGB wurde vereinzelt bereits angewendet, ${ }^{209}$ allerdings ist das Kontakt- und Rayonverbot (noch) nicht in die Strafurteilsstatistik des Bundes aufgenommen worden. ${ }^{210}$

Das Verbot ist auf Antrag der Vollzugsbehörde um jeweils maximal fünf Jahre verlängerbar, ${ }^{211}$ und der Vollzug desselben kann mittels elektronischer Fussfessel überwacht werden. ${ }^{212}$ Ausserdem ist die Verletzung eines Kontakt- und Rayonverbots (wie beim Tätigkeitsverbot) zusätzlich mit Vergehensstrafe nach Art. 294 Abs. 2 StGB bedroht.

\footnotetext{
206 Vgl. vorne IV.2.

207 AS 2014 2055, 2057.

208 Vgl. Botschaft, BBl 20128819 (Fn. 22), 8853.

209 Siehe bspw. Urteil des Obergerichts Zürich SB160374 vom 12. Januar 2017, Dispositivziff. 6. Das Kontaktverbot wurde dabei jedoch nicht angefochten und deshalb in den Erwägungen nicht behandelt.

210 Stand 13. Februar 2018.

211 Art. $67 b$ Abs. 5 StGB.

212 Art. $67 b$ Abs. 3 StGB.
}

\section{a) Anwendbarkeit bei bedingtem Strafvollzug}

103 Die Anwendbarkeit des Kontakt- und Rayonverbots bei bedingtem Strafvollzug ist grundsätzlich gleich zu beurteilen wie beim Tätigkeitsverbot. ${ }^{213}$ Das Anliegen beim Erlass von Art. $67 b \mathrm{ff}$. StGB war primär, ein Kontakt- und Rayonverbot auch unabhängig von einer Probezeit verhängen zu können. ${ }^{214}$ Dies steht einer kumulativen Anwendung aber nicht grundsätzlich entgegen. Die Möglichkeit des Kontakt- und Rayonverbots über Weisungen wird zwar ausdrücklich erwähnt, insbesondere Art. 67c Abs. 3 StGB spricht aber wieder dafür, dass eine Anwendung bei bedingten Strafen vorgesehen war. ${ }^{215}$

104 Wichtig ist aber, dass auch hier die Massnahme im Endeffekt prognoserelevant sein muss. ${ }^{216}$ Ist sie dies nicht, gerät man in einen Prognosewiderspruch, denn ist die Prognose ohne Massnahme genügend gut, kann letztere nicht erforderlich sein. Den Vorwurf des gesetzlich nicht vorgesehenen Strafaufschubs zugunsten einer Massnahme muss man sich wohl auch hier gefallen lassen. Auch führt die Anwendbarkeit bei bedingtem Strafvollzug wiederum zu erheblichen Abgrenzungsschwierigkeiten zu den entsprechenden Weisungen bezüglich Aufenthalt.

\section{b) Verhältnis zur Weisung bezüglich Aufenthalt}

${ }^{105}$ Das Obergericht Zürich hatte einen Fall zu beurteilen, der sich kurz vor Inkrafttreten des Kontakt- und Rayonverbots

\footnotetext{
213 Vgl. vorne IV.2.a).

214 Botschaft, BBl 20128819 (Fn. 22), 8852.

215 Botschaft, BBl 20128819 (Fn. 22), 8852 und vorne IV.2.a).

216 Vgl. vorne II.2.d).
} 
(Art. $67 b$ StGB) ereignet hatte. Da der teilbedingte Vollzug einer Freiheitsstrafe gewährt wurde, erteilte das Gericht ein Kontakt- und Rayonverbot in Form einer Weisung. ${ }^{217}$ Eine Weisung mit diesem Inhalt war also schon vor Inkrafttreten der Art. $67 b$ ff. StGB möglich.

106 Grundsätzlich ist festzustellen, dass das Kontakt- und Rayonverbot analog zum Tätigkeitsverbot aus der Weisungskompetenz ausgenommen werden sollte. ${ }^{218}$ Sind die Voraussetzungen für ein Kontakt- bzw. Rayonverbot nach Art. 67b StGB erfüllt, dann kann dieses angeordnet werden. Sind die Voraussetzungen nicht erfüllt, weil das Kontaktverbot bspw. generell nicht verhältnismässig ist, dann ist nicht ersichtlich, wieso selbiges dann als Weisung verhältnismässig sein soll. Das Kontakt- und Rayonverbot sieht - anders als das Tätigkeitsverbot - keine Mindeststrafhöhe vor (Art. $67 b$ StGB).

Eine Unterscheidung nach dem Ziel des Kontaktverbots (zum Schutz Dritter oder im Interesse des Verurteilten) würde ebenfalls zu Umgehungen einladen und lässt sich je nach Bedarf leicht behaupten, weshalb auch dies kein geeignetes Kriterium darstellt. ${ }^{219}$ Der Bundesrat führte zum Kontakt- und Rayonverbot auch aus, dass dieses "am ehesten in Situationen sinnvoll [ist], in denen es um den Schutz einzelner konkreter möglicher Opfer geht». ${ }^{220}$

217 Siehe Urteil des Obergerichts Zürich SB150260 vom 2. Oktober 2015, E. 2.1.1 ff.

218 Vgl. vorne IV.2.b).

219 Vgl. die Ausführungen zum Tätigkeitsverbot vorne IV.2.b); a. M. BSK StGB-Schneider/Garré (Fn. 12), Art. $44 \mathrm{~N} 27$.

220 Botschaft, BBl 20128819 (Fn. 22), 8853.
108 Fraglich ist, ob die Weisung bezüglich Aufenthalt das mildere Mittel darstellt und deshalb in Konkurrenz zum Kontakt- und Rayonverbot nach Art. 67b StGB weiterbestehen soll. Als Argument liesse sich hier wiederum die Strafandrohung nach Art. 294 StGB anführen. Auch sieht Art. $67 b$ StGB keine Mindeststrafe vor. Wird ein Kontakt- und Rayonverbot nach Art. $67 b$ StGB zusammen mit dem bedingten Strafvollzug angeordnet, führt das Bestehen der Probezeit nicht automatisch zur Aufhebung des Verbots (Art. $67 b$ Abs. 4 StGB).

109 So ist aus Sicht eines Beschuldigten aktuell sicher wünschenswert, dass er «nur» eine Weisung erhält und keine mit Freiheitsstrafe bedrohte Massnahme, die allenfalls über die Probezeit hinauswirkt. Im Gegensatz zum Tätigkeitsverbot kann ein Kontakt- und Rayonverbot einen bloss geringfügigen Eingriff darstellen, wenn bspw. der Kontakt zur Exfrau verboten wird und keine gemeinsamen Kinder vorhanden sind. ${ }^{221}$ Es besteht deshalb durchaus ein sinnvoller und vertretbarer Anwendungsbereich für Weisungen bezüglich Aufenthalt und Kontakt. Dies bedeutet aber im Ergebnis, dass das Gericht bei bedingtem Strafvollzug zu begründen hat, weshalb es ein Kontaktoder Rayonverbot nach Art. $67 b$ StGB für notwendig erachtet und eine entsprechende Weisung nicht ausreicht. Auch sollte man sich davon verabschieden, bei Weisungen den Resozialisierungsgedanken in den Vordergrund zu stellen.

11 In der Literatur wird teilweise mit dem Gedanken gespielt, Electronic Monitoring auch bei geeigneten Weisungen zur

221 Vgl. Urteil des Obergerichts Zürich SB150485 vom 10. Mai 2016, Dispositivziff. 6. 
Anwendung zu bringen, wie dies bspw. nach Art. $67 b$ Abs. 3 StGB für das Kontakt- und Rayonverbot möglich ist.222 Davon ist jedoch Abstand zu nehmen, denn es handelt sich um einen schweren Eingriff in die Privatsphäre und die persönliche Freiheit, welcher den Rahmen von Weisungen sprengt. Der Gesetzgeber hat die elektronische Überwachung nicht als Begleitmassnahme des bedingten Strafvollzuges vorgesehen. ${ }^{223}$ Es bestünde ausserdem das Risiko, dass der bedingte Vollzug dann schlicht zur unbedingten Strafe, vollzogen in Form der elektronischen Überwachung nach Art. $79 b$ StGB224, verkommt.

\section{Fazit}

111 Auf die Frage nach dem Verhältnis von Weisungen und Massnahmen gibt es keine allgemeingültige Antwort. Die Abgrenzung muss für jede Massnahme einzeln vorgenommen werden.

Zusammenfassend kann festgestellt werden, dass sich die ambulante Massnahme und ihr Weisungspendant (bzw. der bedingte Strafvollzug) gegenseitig ausschliessen. Eine Weisung betreffend ärztliche oder psychologische Betreuung ist nur dann gerechtfertigt, wenn die beschuldigte Person ihr Einverständnis gibt.

222 Bspw. bei Benjamin Meier, Der Fussballfan - ein Gewalttäter?, Diss. Zürich, Basel 2016, 296, jedoch nur am Rande erwähnt; unkritisch auch MüKo-Gross (Fn. 71), § 56c N 36.

$223 \mathrm{Vgl}$. Botschaft vom 4. April 2012 zur Änderung des Strafgesetzbuchs und des Militärstrafgesetzes (Änderungen des Sanktionenrechts) (BBl 2012 4721), $4721 \mathrm{ff}$.

224 Art. $79 b$ StGB und damit das Electronic Monitoring als Vollzugsform ist am 1. Januar 2018 in Kraft getreten, AS 2016 1249, 1253 f.
113 Das Tätigkeitsverbot regelt die Möglichkeiten eines vollständigen oder teilweisen Verbots eines Berufs bzw. einer Tätigkeit abschliessend. Aufgrund der Schwere des Eingriffs in die Wirtschaftsfreiheit besteht neben dem Verbot nach Art. 67 StGB kein Anwendungsbereich für eine inhaltsgleiche Weisung.

114 Da das Kontakt- und Rayonverbot gerade auch für den Bereich der leichten und mittleren Kriminalität geschaffen wurde und sich auf einen geringfügigen Eingriff in die persönliche Freiheit beschränken kann, spricht grundsätzlich nichts gegen eine Erteilung als Weisung. Dies führt dazu, dass bei bedingten Strafen ein Konkurrenzverhältnis zwischen dem Kontakt- und Rayonverbot nach Art. $67 b$ StGB und der entsprechenden Weisung besteht. Eine Anordnung der Massnahme im Falle des bedingten Strafvollzugs muss also unter Einbezug der Weisungsmöglichkeit explizit begründet werden.

115 In weitere Überlegungen wäre allenfalls das Strafbefehlsverfahren einzubeziehen, da der Staatsanwaltschaft dort kein Kontakt-, Rayon- oder Tätigkeitsverbot als Massnahme zur Verfügung steht. 